\title{
Hufelands Beitrag zur Bäderheilkunde
}

Empirismus und Vitalismus in seinen balneologischen Schriften

Von Markwart Michler

\section{Einleitung}

Christoph Wilhelm Hufeland ist der Medizingeschichte als bedeutender Seuchen- und Sozialhygieniker, als Reformator des Gesundheitswesens und des Ärztestandes im Gedächtnis geblieben. Auch der Verfasser der Makrobiotik, jenes Werkes, das um die Wende zum 19. Jahrhundert in der Gesundheitserziehung Epoche machte, ist bis heute unvergessen. Seine Bedeutung für die Balneologie scheint darüber fast gänzlich in den Hintergrund getreten zu sein, und selbst die Geschichtsschreibung der Bäderheilkunde wußte schon am Anfang unseres Jahrhunderts seine Verdienste kaum noch zu würdigen ${ }^{1}$.

Dennoch hat Hufeland der Balneologie sein Leben lang ein gleichbleibendes Interesse entgegengebracht und Entscheidendes zur Entwicklung der Badeorte und zur Einrichtung zweckmäßiger Kuranlagen beigetragen. Dabei sind Badehygiene, Hydrotherapie und Balneologie von ihm gleichermaßen gefördert worden, wenngleich er zwischen allgemeiner Wasseranwendung und der Verordnung der Heilquellen einen grundsätzlichen Unterschied gewahrt wissen wollte. Bei einem Mann, der zugleich als Erzieher auf die Ärzte seiner Zeit maßgeblich eingewirkt hat, muß daher die Frage nach seinem Beitrag zur Bäderheilkunde von wesentlichem Interesse sein, läßt sich doch voraussetzen, da $\beta$ er auch darin einen entscheidenden Einfluß auf seine Zeitgenossen ausgeübt hat.

Wie in den Fragen der Lebensverlängerung, so hat er sich auch für den Gebrauch der Bäder zugleich als ärztlicher Aufklärer und als Verfasser wissenschaftlicher Schriften eingesetzt: Noch als Hofmedicus in Weimar veröffentlichte er 1790 in Bertuch's Journal des Luxus und der Moden seinen Aufruf zur Wiedereinführung der Bäder in Deutschland, der freilich zunächst der Erneuerung der Badehygiene galt ${ }^{2}$. Schon früher hatte er in dieser Zeitschrift auf die Bedeutung regelmäßiger Bäder als Schönheitsmittel aufmerksam gemacht ${ }^{3}$, und auch in den folgenden Jahren empfahl er ihren Gebrauch in seinen gesundheitspädagogischen Schriften ${ }^{4}$. Als sein Lehrer Lichtenberg 1793 seinen Aufsatz «Warum hat Deutschland noch kein großes 
öffentliches Seebad ?» veröffentlichte, wiederholte er dessen Aufforderung in den Neuesten Annalen der französischen Arzneikunde ${ }^{5}$. Auch in seiner Makrobiotik widmete er dem Baden in der See bereits einen eigenen Abschnitt und bezeichnete es hier "(ohngeachtet seiner großen Heilkräfte in Krankheiten) dennoch als das naturgemäßeste Hülfsmittel, auch blos zur Erhaltung und Befestigung der Gesundheit $\gg .^{6}$ Bereits in diesen, vorwiegend an einen breiten Leserkreis gerichteten Schriften hat er also ganz allgemein auf die Heilkraft des Wassers hingewiesen ${ }^{7}$, obwohl er mit hydrotherapeutischen Publikationen erst in seinen späteren Lebensjahren hervorgetreten ist $^{8}$.

\section{Hufelands balneologische Schriften}

Auch die Bedeutung der Heilquellen war Hufeland frühzeitig nahegebracht worden: Bereits der eigene Vater hatte sein Interesse geweckt, die wissenschaftliche Grundlage aber gewann er aus den Schriften von Friedrich Hoffmann, ihm blieb er auch in seiner späteren Arbeit verpflichtet und hat ihn dankbar als «wissenschaftlichen Instaurator» der Bädertherapie anerkannt $^{9}$. Zudem hatte er als junger Praktiker die Böhmischen Bäder besucht und hier zum erstenmal Gelegenheit zu eigener Beobachtung gehabt ${ }^{10}$. Als er daher 1795 als Professor in Jena das Journal der praktischen Arzneikunde und Wundarzneikunst gründete, das in der Folge die meistgelesene Zeitschrift unter den deutschen Ärzten wurde ${ }^{11}$, da zählt er im Geleitwort unter den publizistischen Aufgaben der neuen Zeitschrift auch «Bemerkungen über die mineralischen Wasser» auf: «Es scheint mir», so schreibt er hier, «als habe man bisher diese höchstwichtige Klasse der Mittel immer noch zu sehr blos physisch und chemisch und zu wenig practisch behandelt, und es würde gewiß ein Vortheil für die Wissenschaft seyn, wenn sowohl Brunnenärzte, als auch andere, die ihre Kranken davon Gebrauch machen lassen, ihre Beobachtungen über das Verhältnis dieser Mittel zum menschlichen Körper sowohl im gesunden, als kranken Zustand, über die Kräfte und beste Anwendungsart derselben in bestimmten Krankheiten, ferner jährliche Listen von der Zahl der Brunnengäste, ..., durch diesen Weg mittheilen wollten. Es wird dies auch die beste Gelegenheit seyn, neuentdeckte Quellen mit ihren Heilkräften bald allgemein bekannt zu machen ».12

In der Tat hat das Journal dann über die Dauer seines Bestehens laufend balneologische Berichte, Krankheitsbeobachtungen und Statistiken veröffentlicht, von denen ein ansehnlicher Teil aus der Feder seines Heraus- 
gebers stammte. Schon im ersten Band berichtet er über die neue Badeanlage in Limmer, die er bei seinem Aufenthalt in Hannover mehrfach besucht hatte. Da die Quelle erst wenige Jahre zuvor in Gebrauch gekommen war, beschreibt er zunächst ihre chemisch-physikalische Beschaffenheit und versucht so bereits zu einer gewissen Abgrenzung der Heilanzeigen zu gelangen. Schließlich nennt er die Anzahl der durchgeführten Kuren in den letzten beiden Jahren und schildert die bereits bestehenden und für die Zukunft geplanten Badeanlagen. Der systematisch abgefaßte Bericht will dem praktischen Arzt eine klare Vorstellung von den Wirkungen und Möglichkeiten dieses Bades vermitteln, und so vergißt er am Ende auch nicht den Hinweis, daß die Kurmittel an Bedürftige unentgeltlich abgegeben werden ${ }^{13}$.

Ein besonderes Gewicht erhalten diese Aufzeichnungen durch die damals nicht selbstverständliche Tatsache, daß sie aus eigener Anschauung gegeben sind, und diesen Grundsatz behält Hufeland auch in der Folgezeit bei. So entstehen seine «Flüchtigen Reisebemerkungen », die er zum erstenmal von einer Reise im Sommer 1788 veröffentlicht, und denen in späteren Jahren weitere Berichte folgen ${ }^{14}$. Zunächst berichtet er hier von der neuen «Trinkund Badeanstalt » in Sagard auf Rügen und rühmt den örtlichen Vorzug der nahen See, die gleichzeitig Gelegenheit zu Seebädern gibt ${ }^{15}$.

Doch Hufeland hatte seine Fahrt nicht allein zu Studienzwecken angetreten: Durch eine schwere Erkältung, die er sich 1797 anläßlich eines Krankenbesuches bei naßkaltem Novemberwetter zugezogen hatte, war er über Nacht auf dem rechten Auge erblindet ${ }^{16}$. Seine Jenenser Kollegen behandelten ihn erfolglos, warnend stand das Schicksal seines mit dreiundfünfzig Jahren erblindeten Vaters vor ihm, und so beschloß er, ein halbes Jahr lang seine Augen gänzlich zu schonen. Wir wissen aus seiner Selbstbiographie, daß er in der Hoffnung auf Heilung von Rügen in das erste deutsche Seebad Doberan weiterreiste, dessen Gründung durch den Rostocker Professor Vogel er soeben in seiner Makrobiotik begrüßt hatte ${ }^{17}$. Doch Doberan brachte keine sichtliche Besserung seines Zustandes und vor allem keine Gewißheit über die Erhaltung der Sehkraft auf dem anderen Auge. So reist er weiter nach Kiel und nachdem er hier «einen der vorzüglichsten Augenärzte Teutschlands » - ebenfalls vergeblich - konsultiert hatte, entschließt er sich zu einer zweiten Kur in Pyrmont. Diese muß noch weniger günstig verlaufen sein, denn am Ende seiner Reisebemerkungen lesen wir, daß ihn ein Fieber daran gehindert habe, «die naheliegenden Bäder Driburg, Nenndorf und Meyenberg zu besuchen ${ }^{18}{ }^{18}$ Hufelands eigener Erkrankung verdanken wir 
daher auch seinen Bäderbericht, der noch die einzelnen Stationen seines Weges widerspiegelt, und es ist bemerkenswert, wie günstig er trotz der Erfahrungen am eigenen Leib über beide Bäder berichtet ${ }^{19}$. Über den Kurverlauf in Doberan bemerkt er sogar: «Ich selbst befand mich in Betreff einer Schwäche des einen Auges, weswegen ich hieher kam, durch die Anwendung des kühlen Seebads und der Douche von Seewasser ungemein gebessert». Doch dürften sich diese Worte wohl eher auf seinen Allgemeinzustand bezogen haben ${ }^{20}$.

1801 folgte Hufeland einem Ruf nach Berlin, wurde Leibarzt der Königlichen Familie, Direktor des Collegium Medicum und erster Arzt der Charité. Im Sommer des Schicksalsjahres 1806 weilt er dann abermals in Pyrmont und schreibt von dieser Reise, sie sei «ein ganz besonders beglückender und meine geschwächte Gesundheit stärkender Zeitraum» gewesen ${ }^{21}$. Dennoch ist er dieses Mal nicht als Kurgast, sondern als ärztlicher Betreuer der Königin Luise gekommen, von deren Kur er an anderer Stelle sagt, daß sie ihr «neues Leben und die Kraft gab, die schrecklichen Schicksale der folgenden Zeit zu ertragen $\gg .{ }^{22}$ Er nutzt seinen Aufenthalt zu einem Besuch von Meinberg und reist später nach Nenndorf weiter, um hier einer Hautkrankheit wegen selbst die Kur zu gebrauchen. Über Meinberg berichtet er noch im nämlichen Jahr in seinem Journal und bezeichnet es gemeinsam mit Driburg und Pyrmont als «Sprößling eines kraftvollen Stammes, nur in gemilderter Kraft». Meinbergs Quelle nennt er kohlensauer salinisch eisenhaltig und reiht sie damit in die Gruppe von Pyrmont und Driburg ein. Allerdings sei sie von milderer Wirkung als ihre beiden Schwestern und daher gerade für solche Kranke geeignet, «denen jene Wasser zu stark sind, oder bei denen Geneigtheit zu Blutcongestionen, große Reizbarkeit der Nerven oder kränkliche Lungen große Vorsichten nöthig machen». Auch weist er auf ihren Reichtum an kohlensaurem Gas hin und rät zur Einrichtung eines «pneumatischen Kabinetts», um mit pneumatischen Bädern, Duschen und Injektionen Behandlungsmöglichkeiten für Brustkranke, Schleimflüsse, Hautkrankheiten, Geschwüre und Lähmungen zu schaffen ${ }^{23}$.

Hufeland hat im Laufe dieser Jahre die Balneologie nicht allein durch seine Augenzeugenberichte gefördert, schon 1797 hatte er in seinem Journal einen Hinweis auf «Nenndorfs asphaltische Schwefelquelle» gegeben und die wichtigsten Daten aus dem Buch von Hofrat Schröter zu Rinteln mitgeteilt ${ }^{24} .1802$ kündigte er die Gründung der beiden Seebäder Norderney und Colberg an und veröffentlichte in diesem Zusammenhang eine Vergleichstabelle über den unterschiedlichen Salz- und Mineralgehalt des Nord- 
und Ostseewassers ${ }^{25}$. Anhand von kasuistischen Berichten gab er besonders erfolgreiche Einzelkuren bekannt oder setzte Gegenindikationen fest, auch teilte er die chemische Analyse des Driburger Brunnens durch Westrumb mit und machte den neu entdeckten Selkenbrunnen im Harz bekannt ${ }^{26}$.

Vor allem aber erläßt er im Jahre 1802 seine bekannte «Aufforderung an die Brunnenärzte Deutschlands, besonders Schlesiens, nebst einigen Worten über mineralische Wasser überhaupt ». ${ }^{27} \mathrm{In}$ ihr bittet er die Badeärzte, «die wichtigsten Erfahrungen über die Wirkung ihrer Brunnen in diesem Journale, wo sie am geschwindesten allgemein bekanntwerden, mitzuteilen ». Hier kommt er nun auch auf sein Hauptanliegen zu sprechen: «Ich unterscheide nemlich sehr die chemische (naturhistorische) Kenntnis eines Mineralwassers von der practischen Kenntnis seiner Wirkungen auf den menschlichen Körper. Die erstere gehört allerdings dazu, um es unter allgemeine Klassen bringen zu können. Aber erst die practische Analysis, d.h. die Untersuchung, wie verhält sich das Mittel gegen das Reagens des lebenden Körpers, wie ist seine Wirkung aufs Lebende und im Lebenden, und zwar erst im gesunden Zustande und sodann in den mannichfaltigen anomalischen Verhältnissen des kranken Zustandes ? - giebt dem practischen Arzte eine zureichende und befriedigende Erkenntniß. Und daran fehlt es uns, wie mir es scheint, noch bei vielen unserer Mineralwasser. ${ }^{28}$

Hufeland hatte längst erkannt, daß die chemische Quellenanalyse allein keine sichere Aussage über die Wirkung eines Heilquells gestattete. Seine vitalistische Einstellung ließ ihn am Lebenden ohnehin zwischen chemischphysikalischen und modifizierten animalisch-chemischen Prozessen unterscheiden, sie leitete ihn auch zu der Annahme, daß im Mineralwasser Naturkräfte wirksam sind, die einer chemischen Erkenntnis unzugänglich bleiben ${ }^{29}$. Wie in der Praxis am Krankenbett, so versuchte er deshalb auch in der Balneotherapie durch praktische Erfahrungen festen Boden unter die Füße zu bekommen. «Die genaue Erzählung der Anwendung dieser Mittel auf bestimmte Fälle und ihrer Wirkung», so erläutert er sein Vorhaben, «ihr Verhalten nicht bloß gegen das Specielle des Krankheitszustandes, sondern auch gegen das Individuelle des Kranken, - das ists, was ich wünsche, und wodurch allein diese Klasse der Mittel den Grad von Bestimmtheit exhalten kann, den andere haben, und den sie so sehr verdient. ${ }^{30}$

Die Zustimmung unter den Ärzten kann nicht einhellig gewesen sein, und es zeugt von Hufelands Toleranz, wenn er in seinem Journal auch gegensinnige Ansichten zu Wort kommen ließ, die dem gewöhnlichen Wasser die nämlichen Wirkungen zuschrieben wie den mineralischen Trink- und Bade- 
kuren ${ }^{31}$. In seiner Entgegnung weist er noch einmal darauf hin, es komme ihm "weniger auf die in einem Mineralwasser enthaltenen Stoffe und ihre Quantität als auf die Art ihrer Mischung und Darstellung » an ${ }^{32}$. Eben dieser Satz aber bildete zugleich die theoretische Grundlage, auf der er mit seiner Suche nach spezifischen Heilanzeigen für die einzelnen Bäder fußte.

Eine solche Sammlung balneologischen Wissens mußte zu einer größeren Darstellung drängen. Er schreibt sie in den dunkelsten Jahren seines Lebens in Königsberg: 1808 erscheint sein Werk «Praktische Blicke auf die vorzüglichsten Heilquellen Deutschlands» zunächst im Journal als eine Reihe fortlaufender Aufsätze, 1815 wird es dann vermehrt und ergänzt als Buch herausgegeben, nun unter dem Titel: Praktische Übersicht der vorzüglichsten Heilquellen Teutschlands nach eigenen Erfahrungen ${ }^{33}$. Weist die Überschrift der Aufsatzreihe bereits auf den praktischen Nutzen des Werkes hin, so deutet der Buchtitel dann auch ausdrücklich dessen empirische Grundlagen an. Sinngemäß bemerkt er im Vorwort zur Buchausgabe: «Ich habe ferner nichts gesagt, was ich nicht durch eigne Erfahrungen erprobt und bestätigt hätte ${ }^{34}$ und um ihren praktischen Wert zu erhöhen, gibt er seiner Schrift «Zusätze eines Ungenannten» bei, die von einem «der ersten, ältesten und erfahrungsreichsten Praktiker» stammen ${ }^{35}$.

Hufelands Werk ist daher auch nach praktischen Gesichtspunkten gegliedert und handelt die einzelnen Bäder oder zusammengehörende Bädergruppen in gesonderten Kapiteln ab. Ihnen sind «allgemeine Bemerkungen über Mineralwasser und ihren Gebrauch» vorangesetzt, die ein besonderes Interesse verdienen, weil ihr Autor hier seine theoretischen Lehren und allgemeinen Ansichten zur Bäderheilkunde wiedergibt ${ }^{36}$. Zunächst sucht er zu rechtfertigen, warum gerade er, obwohl er doch kein Badearzt ist, ausführlich über die Bäder schreibt und gibt zu bedenken, daß «das Zeugnis eines entfernten Arztes, über den Werth und die Eigenschaften eines Brunnens in mancher Hinsicht beweisender und belehrender ist, als das des gegenwärtigen Brunnenarztes; einmal, weil bei ihm ... jeder Verdacht der Partheilichkeit wegfällt, zweitens weil er die Wirkungen des Heilquells weit mehr in ihrer Verbindung mit entfernten Ursachen und Folgen ... erkennen kann, als der Brunnenarzt, der gewöhnlich nur die unmittelbaren Wirkungen, und also nur ein Fragment der ganzen Kur sieht»».

Hufeland will hier also keine ausführliche Abhandlung über die einzelnen Mineralbrunnen liefern, sondern zunächst die allgemeinen Wirkungen der Bädertherapie und die Grundsätze ihrer Anwendung beschreiben, und anschließend das «eigenthümliche jedes Heilquells, seinen spezifischen Cha- 
rakter» herausheben, um dem praktischen Arzt, der die Bäder nicht aus eigener Erfahrung kennt, die Auswahl zu erleichtern. Deshalb wendet er sich auch gegen die herabsetzenden Machtsprüche mancher neuen Systematiker, die «den Werth der Mittel mehr nach hypothetischen Prämissen, als nach Erfahrung würdigen ».

Doch er wendet sich gleichzeitig auch gegen die überhöhten Ansprüche der Chemie und ihrer Analysen: Zwar findet er die chemische Einteilung der Mineralwasser zur naturhistorischen Übersicht sehr zweckmäßig, doch ist er andererseits überzeugt, «daß die blos chemische Kenntnis die Sache keineswegs erschöpft, und keineswegs dazu hinreicht, den Werth und die besondere Wirksamkeit eines Körpers auf den lebenden Organismus vollkommen und genau zu bestimmen, sondern daß dieses blos durch Versuche am Lebenden selbst ausgemittelt werden kann, und daß also diese allein den Namen einer praktischen, d.h. dem. Heilkünstler in der Behandlung des Lebenden brauchbaren, Kenntnis verdient». Deshalb vermag er auch die künstlichen Mineralwässer nicht den natürlichen gleichzusetzen; denn einmal dürfte man sich nicht anmaßen, ihre Bestandteile wirklich vollständig zu kennen, zum anderen aber «liegt schon in der Innigkeit der Mischung ein so auffallender Unterschied, daß die flüchtigen Bestandtheile ... ungleich schneller von dem künstlichen, als natürlichen Mineralwasser entweichen, welches nothwendig auch bei der Wirkung im Organismus einen bedeutenden Unterschied machen muß». «Das künstliche Selterswasser», so fährt er fort, «ist gewiß ein höchst schätzbares kohlensaures Wasser, aber Selterswasser ist es nicht. Ebenso ist das, nach der chemischen Analyse zusammengesetzte Karlsbader-Wasser ein recht brauchbares medizinisches Laugenwasser, aber kein Karlsbader-Wasser. Man lasse das erstere einige Wochen trinken, und man wird gewiß Atonie des Verdauungs- und allgemeinen Systems erfolgen sehen. Das Karlsbader-Wasser kann man hingegen Monate lang trinken, ohne diese Folgen, ja mit zunehmendem Appetit und Kraft. Beweis genug, daß letzterem etwas beiwohnt, was ersterem fehlt, und was die nachtheilige Wirkung des Laugensalzes wieder aufhebt.»

So kommt er zu dem Schluß, «daß die Mineralwasser eine große, ganz eigene Klasse von Naturproducten und Agentien darstellen », und die Chemie noch lange nicht in der Lage ist, über ihre eigentliche Mischung und ihren Wert zu entscheiden. Er wiederholt daher hier die Frage, ob es nicht hinsichtlich der Wirkung viel mehr auf die Form und Mischung als auf die Grundstoffe selbst ankomme, und fragt weiter: «Kann man im eigentlichen Verstande sagen, man kennt einen Körper in seinem lebendigen Seyn, wenn 
man sein Skelet kennt? - und mehr oder weniger sind alle chemischen Analysen, die doch immer nur erst Producte von Zersetzungs- d.h. Tödtungsprocessen sind, nichts weiter. Denn nicht blos die organischen Körper, sondern auch die unorganischen haben ihr lebendiges Seyn.»

Diese Einbeziehung der unbelebten Natur in seine vitalistische Anschauungsweise ist bei den Heilquellen besonders ausgeprägt und erklärt auch im folgenden erst seinen Rat, die Mineralwasser unmittelbar aus der Quelle zu gebrauchen, d.h. «aus den lebendigen Händen der Natur». Nur so genieße man dieses Naturprodukt «in seiner vollen Kraft und Reinheit», denn jeder Augenblick der Verzögerung sei «mit einem großen Verluste der Heilkraft verbunden », und in der Entfernung gebraucht, müsse ein Mineralwasser nicht allein als ein schwächerer, sondern auch selbst in der Wirkungsart mehr oder weniger veränderter Körper betrachtet werden ${ }^{37}$. Eine solche Auffassung von den eigentümlichen Kräften der Mineralwässer hatte Hufeland bereits in seiner «Aufforderung an die Brunnenärzte Deutschlands» angedeutet, sie macht schließlich auch die Sätze im Vorwort zu seiner Buchausgabe verständlich, man möge sein Werk «als einen Zoll des Dankes ansehen, den ich dieser großen, ganz eigenthümlichen, und nach ihrem wahren Werth noch lange nicht genug erkannten und gewürdigten, Klasse von Naturprodukten abtrage, für so vieles Herrliche, was sie schon der leidenden Menschheit erzeigt, für so manchen Dienst, den sie auch mir in meiner praktischen Laufbahn geleistet haben ${ }^{38}{ }^{38}$ Im Gegensatz zur rationellen Empirie seiner balneologischen Praxis fußt deren theoretische Begründung mithin auf vitalistischen Spekulationen, die später noch eingehend erörtert werden müssen.

In der Einteilung der Mineralwässer hält sich Hufeland freilich noch immer an Friedrich Hoffmann, und es bleibt bei einer Unterscheidung von eisenhaltigen, schwefelhaltigen, laugensalzigen, kochsalzigen und bittersalzigen Wässern ${ }^{39}$. Seine Baderegeln nennt er eine «Bestimmung des Brunnengebrauchs nach den Grundsätzen der neuern, aber auf Erfahrung gegründeten Heilkunde».40 Dennoch stellen sie eine Mischung aus seinen theoretischen Lehren und allgemeinen balneologischen Erfahrungen dar: Danach bildet jedes Mineralwasser nicht nur einen «reizenden Arzneistoff », sondern auch einen Gegenstand der Verdauung, dessen Schwerverdaulichkeit mit der Menge der festen Bestandteile und mit der Art der gelösten Stoffe in der Reihenfolge: Salze, Schwefel, Erden und Eisen steigt. Eine sachgerechte Diät bildet daher die Voraussetzung für eine erfolgreiche Kur, sie muß das Verdauungssystem in seinem «Kampf mit einer rohen Natur» 
unterstützen. Jede Brunnenkur muß aber auch als eine «künstliche Krankheit» betrachtet werden, da sie im Organismus «erhöhte Reizbarkeit, verminderte Kraft, vermehrte Bewegungen des Gefäßsystems bis zu Fieber, Congestionen, sowie Vermehrung oder Hemmung der Ausscheidungen und Absonderungen » verursacht; die Behandlung hat daher solche Zwischenfälle zu berücksichtigen. Schließlich ist jede Brunnenkur zugleich ein «chemisch-animalischer Proze $\beta$ » und bringt «wichtige Veränderungen in der organischen Mischung sowohl zur Bindung, als zur Zersetzung der Materien » hervor. All diese Vorgänge gemeinsam bewirken schließlich den «organischen Heilungsproze $\beta$ » als ein eigenes vitales Geschehen, das auch nach Beendigung der Kur noch fortdauert, erst an seinem Ende zur «wirklichen Krise » führt und daher ärztliche Behandlung und Beobachtung auch in der Zeit der Nachwirkung erforderlich macht.

In seinen leitenden Prinzipien herrschen also theoretische Spekulationen noch immer vor, sobald aber Hufeland auf die eigentliche Art der Brunnenanwendung zu sprechen kommt, gewinnen die praktischen Erfordernisse die Oberhand: Er unterscheidet die starke, die mittlere und die kleine Trinkkur, behält die starke und die kleine Kur aber nur einzelnen Krankheiten vor und verordnet bei der mittleren die noch immer erhebliche Trinkmenge von vier bis zu maximal zwölf Bechern pro Tag. Starke und mittlere Kur dürfen freilich nur in den Sommermonaten gebraucht werden, weil der Organismus in der Kur reizbarer und empfänglicher für Erkältungen ist, die kleine läßt sich dagegen «zu jeder Jahreszeit und mitten im härtesten Winter» durchführen. In der Regel sollte der Brunnen am frühen Morgen unmittelbar nach dem Aufstehen getrunken werden. Die folgenden drei bis vier Stunden bilden die Verdauungszeit und sind daher der Hauptzweck des Aufenthalts: Ruhe ohne Schlaf ist deshalb die Kurregel für den Vormittag. Ausführliche Anweisungen sind der Brunnendiät gewidmet, sie umfaßt genaue Ernährungs- und Bekleidungsvorschriften und ein «Regimen der Seele», das die «Anstrengung der Denkkraft» und die «Leidenschaft» auszuschalten hat ${ }^{41}$.

Eine Kombination von Brunnenkur und Arzneimittelgaben lehnt er ab, denn einmal heile die Kraft der besten Mineralwässer erfahrungsgemäß einen Teil jener Krankheiten, die der Gebrauch der kräftigsten Arzneimittel nicht zu heilen vermochte, zum zweiten aber kenne man ihre Wirkung so wenig, daß ihr Verhältnis zu anderen einwirkenden Stoffen keineswegs genau zu bestimmen sei. Es bestehe daher die begründete Sorge, daß man den chemisch-animalischen Operationen, die sie im Organismus auslösten, ent- 
gegenwirke. Lediglich die Gabe von Laxantien sowie von verdauungsfördernden und krampfstillenden Medikamenten sei erlaubt, bei hartnäckigen Übeln ausnahmsweise auch die Unterstützung der Brunnenwirkung durch angemessene Arzneistoffe, so z.B. durch sulphurische Mittel bei Schwefelbrunnen oder durch alkalische bei alkalischen Wässern. Mögen die letzten Konzessionen von chemischen Vorstellungen ausgehen, so dürfte sein striktes Verbot des Opiums und des Alkohols von seinem Kampf gegen den Brownianismus mitbestimmt $\operatorname{sein}^{42}$. Um so eindringlicher befürwortet er in geeigneten Fällen die gemeinsame Anwendung von Bade- und Trinkkur, insbesondere bei den Stahlbädern hält er sie für sehr wesentlich. Auch die Lokal- und Duschbäder bezeichnet er als eine «vorzügliche Errungenschaft der neueren Heilkunst ».43

Am Ende dieses allgemeinen Teils geht er schließlich auf die absoluten Kontraindikationen gegen eine Badekur ein. $\mathrm{Zu}$ ihnen rechnet er sämtliche Lungenerkrankungen (mit Ausnahme der kohlensauren muriatischen Quellen), die Eiterung innerer Eingeweide (ausgenommen die der Harnwege und oberflächliche Schleimhauteiterungen), hektische Fieber, Brust- und Bauchwassersucht sowie Schwangerschaft wegen der Abortgefahr ${ }^{44}$.

War die Einführung des Werkes nicht unwesentlich von Hufelands theoretischen Anschauungen bestimmt, so zeigen die folgenden Kapitel über die einzelnen Badeorte, wie sehr er sich um klare Heilanzeigen auf der Grundlage praktischer ärztlicher Erfahrungen bemühte. Zunächst versucht er den Grundcharakter einer Quelle zu bestimmen und leitet aus ihm ihre Wirkung ab. So nennt er beispielsweise Pyrmont und Driburg in der Terminologie seiner Zeit « excitierend, erhitzend, das Blut nicht allein bewegend, sondern in seinem rothen balsamischen Theile vermehrend und erwärmend, tonisch stärkend und zusammenziehend $»{ }^{45} \mathrm{Karlsbad}$ dagegen wirkt reizend auf den Magen- und Darmkanal und auf alle Sekretionsorgane, insbesondere auf Leber, Nieren und die Eingeweide des Unterleibs. Es vermehrt daher alle Absonderungen, vor allem die des Darms. Ferner wirkt es auf das Blutsystem und erhöht die lymphatische Tätigkeit, doch verursacht es dabei im Gegensatz zu den Stahlwässern ein Nachlassen der reizenden Erregung ${ }^{46}$. Aus diesen empirisch festgestellten Wirkungen leitet er schließlich die Indikationen und Gegenanzeigen ab und empfiehlt Pyrmont und Driburg gegen Blutarmut und bei starken Blutverlusten, nach schweren hitzigen Erkrankungen und «übermäßigen Anstrengungen der Seelenkräfte» sowie bei Nervenkrankheiten, Nervenschwäche und folgerichtig auch bei der Hypochondrie, die auf der Grundlage einer allgemeinen Schwächung der Nerven 
entsteht. Zu Karlsbad dagegen rät er bei hartnäckigen Gelbsuchten und Kachexien, bei Lebererkrankungen, Obstipation und bei jenen Formen der Hypochondrie, die durch Überfüllung der Visceralorgane ausgelöst werden; ferner bei Nieren- und Blasensteinen, denn «das Carlsbader Wasser ist das kräftigste steinauflösende und steinabtreibende Mittel, das ich bisher kenne ${ }^{47}$

Das letzte Kapitel des Werkes handelt vom Seebad, doch betrachtet es Hufeland hier nicht unter dem Gesichtspunkt der Gesundheitsprophylaxe, sondern versucht seine Heilanzeigen bei ernsthaften Erkrankungen abzugrenzen. Seine allgemeine Wirkung beschreibt er als «reizend und belebend, zunächst für die Haut, demnächst aber für das ganze Nerven-, Lymph- und Drüsensystem und die Secretionsorgane ».48 Neben Rheumatismen, Hautund Drüsenerkrankungen empfiehlt er die See daher vor allem bei Nervenleiden, wie Krämpfen, Migräne und nervösen Zahnschmerzen. Vor allem aber hält er sie bei der Epilepsie und der Chorea für das souveräne Mittel, eine Besserung oder gar Heilung herbeizuführen. Von den beginnenden Lähmungen sagt er freilich, daß er mitunter zwar völlige Heilungen, in anderen Fällen aber eine deutliche Verschlimmerung gesehen habe. Hier zeigt sich nun noch einmal, mit welcher Zielstrebigkeit er auch in der Balneologie zu einer möglichst klaren Indikationsstellung zu gelangen sucht: «Mancher, dem das Seebad nicht geholfen hatte», so schreibt er, «wurde in Pyrmont, und mancher, der Pyrmont ohne Nutzen gebraucht hatte, im Seebade geheilt. - Hier stoßen wir nun auf den wichtigsten, aber auch schwersten Punkt der Untersuchung, - die Hauptsache für den Praktiker - zu bestimmen, in welchem Fall und in welcher Modifikation der Nervenstimmung ist das Seebad passender, und in welchem Pyrmont oder überhaupt ein Eisenbad ?» «Meine Erfahrung hat mich folgendes gelehrt», so fährt er fort, und führt dann aus, daß komplikationslose Krankheitsformen in beiden Bädern den nämlichen Nutzen finden, daß aber erhöhte Reizschwäche des Nervensystems, Nervenschwäche in Verbindung mit Vollblütigkeit oder Neigung zu Entzündungen und Anomalie des Hautnervensystems besser auf das Seebad ansprechen, während bei Nervenschwäche mit Komplikationen am Blutsystem oder am Herzen Pyrmont die besseren Dienste leistet ${ }^{49}$.

Hufeland hat die Mineralquellen einmal die «vorzüglichsten Apotheken » genannt ${ }^{50}$, und die Tatsache, daß auch er noch immer auf Aderlaß, Opium und Brechmittel als die «drei Heroen der Heilkunst» angewiesen blieb ${ }^{51}$, dieser Mangel an spezifisch wirksamen Heilmitteln, macht ein solches Wort 
verständlich. Sein Werk mußte daher als Bereicherung der therapeutischen Möglichkeiten empfunden werden und dem Praktiker von damals ein wertvolles Hilfsmittel an die Hand geben. Mochte es sich auch mit manchen Ansichten an die Vergangenheit anlehnen, so füllte es mit seinem Bemühen um. klare Indikationen doch eine Lücke trotz der mannigfachen Bäderliteratur der Zeit. Überprüft man am Ende noch einmal seine Heilanzeigen für die einzelnen Bädergruppen, dann zeigt es sich, daß sie zu einem großen Teil auch heute noch Gültigkeit besitzen. Auch ohne die zahlreichen balneologischen Berichte in Hufelands Journal würde eine solche Feststellung deutlich machen, welche Fülle an soliden praktischen Erfahrungen seinem Werk zugrundelag. Lediglich die Empfehlung des Seebades bei Epilepsie und Chorea fällt gänzlich aus der Reihe der übrigen Heilanzeigen, und in seinen gesamten Schriften findet sich keinerlei Anhalt, auf welche eigenen Erfahrungen sich diese Heilmaßnahme gründete. Zu ihrer Erklärung bleibt man auf die Lebensbeschreibung seines Schwiegersohnes Alexander de Stourdza angewiesen, wo sich die kurze Bemerkung findet: «Pendant son séjour dans l'ancienne Prusse, dans ces sables du nord que la main de l'homme a rendus fertiles, Hufeland opéra de belles cures. Il aimait plus tard à se rappeler un jeune israelite, qu'il avait guéri de l'épilepsie en lui conseillant l'usage assidu des bains de mer. Ce jeune homme, dix ans après sa guérison, vint le surprendre à Berlin, en lui exprimant sa reconnaissance de la manière la plus touchante. $»^{52}$ Man muß daher wohl annehmen, dieser eine, gewiß eindrucksvolle Fall habe auch für Hufeland noch immer genügt, den Seebädern bei Epilepsie und Chorea als «spezifischem Heilmittel» das Wort zu reden, auch wenn eine so voreilige Empfehlung bei ihm eine Ausnahme bleibt.

Die folgenden Friedensjahre brachten Hufeland die Arbeitslast zahlreicher Staatsämter. Dennoch hat er weiterhin der Balneologie seine Aufmerksamkeit gewidmet. Hatte er schon in seinem Buch über die Heilbäder vor den Spielbanken gewarnt und eine «Brunnenpolizei» empfohlen ${ }^{53}$, so lag es zugleich im Rahmen seiner Bemühungen um die Hygiene, wenn er sich im Journal für eine bessere Einrichtung der Badeanlagen einsetzt, einen einwandfreien Versand der Mineralwasser erstrebt und statistische Erhebungen über deren Verbrauch in Berlin vornimmt ${ }^{54}$. Schon früher hatte er von Gesetzes wegen die Pflicht zur deutlichen Kennzeichnung künstlich hergestellter Brunnen gefordert und eine regelmäßige Revision der Heilquellen vorgeschlagen ${ }^{55}$. Auch die Verwendung eines Badethermometers war von ihm mehrfach angeraten worden ${ }^{56}$. 
In dieser Zeit äußerer Ruhe nimmt er auch seine «Flüchtigen Reisebemerkungen » wieder auf: 1816 sieht ihn Nenndorf erneut als Kurgast, er besucht auch Pyrmont und berichtet über die Fortschritte in den Kur- und Badeanlagen ${ }^{57}$. Als er 1821 abermals in Nenndorf weilt, schließt er eine größere Reise an, auf der er neben Pyrmont nicht nur Meinberg und Eilsen, sondern auch die «Rheinbäder» berührt ${ }^{58}$. In Meinberg findet er die Gasbäder und -douchen verwirklicht, deren Einrichtung er bei seinem ersten Besuch empfohlen hatte. Ausführlich berichtet er über Wiesbaden, das er in seinem Bäderbuch nur kurz gestreift hatte, nennt Schwalbach «unstreitig das lieblichste aller Stahlwasser für den Geschmack » und kennzeichnet Ems als «vortreffliches Surrogat» für jene Fälle, in denen eigentlich Karlsbad angezeigt, aber von zu starker Wirkung wäre. Er beschließt seinen Bericht mit dem Satz: «Im Ganzen ist auf meiner diesjährigen Reise mein Glaube von neuem befestigt worden, daß die einzig richtige Ansicht der Brunnenkuren die ist, die Kur als eine künstlich erregte Krankheit, und die Nachwirkung als die Krise derselben zu betrachten. ${ }^{59}$ Die Worte zeigen, daß er seine Auffassung von der Wirkung der Brunnenkuren auch in späteren Jahren nicht mehr geändert hat ${ }^{60}$.

1823 reist er noch einmal nach Böhmen und Schlesien ${ }^{61}$ und berichtet über die jüngste Entwicklung der schlesischen Bäder, die er 1813 während seines Breslauer Aufenthaltes zum erstenmal näher kennengelernt hatte ${ }^{62}$. OberSalzbrunn empfiehlt er hier ausdrücklich bei der «floriden phthisischen Anlage» und bemerkt: «Man kann rechnen, daß zwei Drittheile der hier befindlichen Kranken Brustkranke sind, und die hustenden Kranken sind hier eben so die herrschende Klasse, wie in Karlsbad die Hypochondrischen, in Pyrmont die Nervenschwachen, in Nenndorf die Hautkranken. - Man kann in dieser Hinsicht mit Recht sagen, jedes wirklich ausgezeichnete Bad hat auch seinen eigenen vorherrschenden Karakter seines Publikums, seine eigene Farbe. ${ }^{63}$ Unter den böhmischen Bädern aber rühmt er in einer ausführlichen Schilderung das junge, erfolgreiche Marienbad mit seinem Kreuzbrunnen und schließt diesen Abschnitt seines Berichtes mit dem Satz: «Eins jedoch muß ich anführen, und was wohl allem übrigen die Krone aufsetzt, daß nehmlich Göthe, der Stolz unserer Nation, im Kreuzbrunnen nach einer schweren Krankheit seine beste Hülfe, und in diesem Sommer die vollkommenste Wiederherstellung gefunden hat.»-Es war das Jahr der Marienbader Elegie ${ }^{64}$.

Auch in den Aufsätzen der späteren Jahre bleibt es Hufelands wesentlichstes Anliegen, zu einer möglichst klaren Abgrenzung der Heilanzeigen zu 
gelangen. Solchem Bestreben dienen seine kasuistischen Mitteilungen, in denen er besonders eindrucksvolle Heilerfolge klar umrissener Krankheitsbilder bekanntgibt ${ }^{65}$. Noch immer weist er freilich auch auf die gute Wirkung der Aachener Quellen bei syphilitischen Erkrankungen hin, nennt er den Salzbrunnen von Eger ein «Auflösungsmittel der Tuberkeln ». ${ }^{66}$ Bis an sein Lebensende setzt er auch die Berichte über neu entdeckte Heilquellen und über den Ausbau der Kuranlagen fort: Er macht Bad Berka und den Ludwigsbrunnen in der Wetterau bekannt ${ }^{67}$, er rühmt die neuen Gas- und Schlammbäder zu Eilsen als die «einzigen ihrer Art̀ in Deutschland $»^{68}$ und weist auf die Einrichtung von «Regen-Tropf-Sturz- und Spritzbädern» in Norderney hin ${ }^{69}$. Dem nämlichen Zweck dienen seine «Badechroniken », die er in einzelnen Bänden des Journals veröffentlicht ${ }^{70}$. Noch im Novemberheft von 1823 gibt er «Frohe Nachricht aus Karlsbad von dem plötzlich wieder entsprungenen Schloßbrunnen », dessen Quelle seit dem 15. Oktober von neuem floß, nachdem sie vierzehn Jahre zuvor plötzlich versiegt war ${ }^{71}$. Eine ausführliche Beschreibung des Egerbrunnens ergänzt schließlich die Angaben über Franzensbad in seinem Buch über die Heilquellen ${ }^{72}$, und ein Vorwort aus seiner Feder sollte Treumanns Schrift über Freienwalde ein stärkeres Gewicht geben, dessen bescheidenem Glanz inmitten einer herben Landschaft noch ein halbes Jahrhundert später Fontane ein Denkmal gesetzt hat ${ }^{73}$. Dabei wußte Hufeland wohl, daß es «nicht zu den großen und vorzüglichen Heilquellen » gehörte, doch er sah, daß es für die weniger bemittelten Kreise Berlins seiner günstigen Lage wegen unentbehrlich war, und so rühmt er auch an anderer Stelle seine «in den Sandebenen der Mark wirklich überraschende Lage ». ${ }^{74}$

All diese verstreuten Aufsätze, Bekanntmachungen und Notizen zeigen zugleich, in wie hohem Maße er auch in der Balneologie immer wieder zum Erzieher der Ärzte geworden war. Besonders deutlich kommt dies noch einmal zum Ausdruck in seiner «Bitte an die Ärzte», die ihre Kranken in Heilbäder schicken ${ }^{75}$ : Hier warnt er die Hausärzte nachdrücklich davor, ihren Patienten spezielle Instruktionen zum Gebrauch der Mineralquellen mitzugeben, die den Badearzt überflüssig machen; denn zur Überwachung einer Kur sei nicht nur die genaue Kenntnis des Brunnens unerläßlich, auch der Wechsel im Befinden des Kranken erfordere eine ständige Betreuung am Ort. Anstelle solcher unangebrachten Ratschläge solle der Hausarzt seinem Kollegen einen Brief zukommen lassen, in dem er eine kurze Charakteristik des Patienten gibt und dessen Krankengeschichte mitteilt. «Dieß allein ist's », so beschließt Hufeland seine Bitte, «was der Hausarzt thun kann, 
und dieß muß er thun, wenn er gewissenhaft ist. Denn seine Kranken geradezu in die Bäder zu schicken, ohne dem dortigen Arzt einen Wink über ihre Krankheit und Eigenthümlichkeit zu geben, ist eben so unverantwortlich, und kann die übelsten Folgen haben.»

Zu Ende des Jahres 1832 gibt er noch einmal einen ausführlichen kasuistischen Bericht über den Nutzen des Wildunger Wassers bei Blasenkrankheiten - es ist seine eigene Krankengeschichte ${ }^{76}$. Nüchtern und sachlich beschreibt er in ihr die akute Harnverhaltung, die er im Juni des vorangegangenen Jahres erlitten hatte, und schildert ihren quälenden, über zwölf Wochen währenden Verlauf - freilich ohne Kenntnis des Grundleidens, einer Prostatahypertrophie, die vier Jahre später zu seinem Tod führen sollte. Nicht ohne Bewegung liest man die einleitenden Zeilen: «Der Verfasser bittet dießmal seine geehrten Leser um die Erlaubnis, sie mit seiner eigenen Person zu unterhalten, da er leider selbst in den Fall gekommen ist, Gegenstand einer nicht uninteressanten pathologischen und therapeutischen Beobachtung zu werden. Er hat eine schwere Leidenszeit durchlebt, und war nahe daran, auf immer von ihnen Abschied zu nehmen. Er hat es, Dank sei der gnädig waltenden Vorsehung, glücklich überstanden, und er hält es nun für seine Pflicht, ihnen das, was bei seiner Leidensgeschichte wissenschaftlich und praktisch merkwürdig war, öffentlich mitzutheilen. » Danach schildert er die Schwierigkeiten des Katheterismus, der selbst Rust und Graefe erst am sechsten Krankheitstage gelang, und berichtet von der hartnäckigen eitrigen Cystitis, die nach Abklingen des akuten Zustandes zurückblieb, mit Abmagerung und starkem Verfall der Kräfte einherging und auf keinerlei Medikamente ansprach. Da erinnerte er sich des Wildunger Wassers, das er in ähnlichen Fällen mit Vorteil verordnet hatte, und nach vierwöchentlichem Gebrauch waren die Beschwerden fast gänzlich gehoben. «Ich fuhr nun fort », so schreibt er weiter, « alle Morgen ein Glas dieses wohlthätigen Wassers zu trinken, und habe dieß das ganze folgende Jahr fortgesetzt, und ihm verdanke ich es, daß ich seit dieser Zeit nicht allein von dem Schleimabgang, sondern auch von den übrigen Blasenbeschwerden befreit bin, und ohne in dem ganzen Jahre etwas anders gebraucht zu haben, in dieser Beziehung das vollkommenste Wohlseyn genossen habe. ${ }^{77} \mathrm{Am}$ Ende versucht er auch hier noch die Heilanzeigen des Wildunger Wassers klar abzugrenzen und spricht von seiner spezifischen Wirksamkeit bei atonischen Blasenkrankheiten, bei der Blasenschleimsucht und der Lithiasis. 
Zweifellos hat Hufeland auch die Bäderheilkunde vorzüglich als ärztlicher Praktiker gefördert, und eine historische Würdigung seiner Verdienste wird zwischen seiner balneologischen Praxis und seinen theoretischen Lehren zu unterscheiden haben. Bereits im Geleitwort zu seinem Journal hatte er die Mineralquellen aus dem Streit der medizinischen Systeme herausgenommen und wieder der praktischen Erfahrung am Kranken zugeführt ${ }^{78}$. Seine Aufrufe und Schriften haben diesen Grundsatz konsequent weiterverfolgt, und es läßt sich voraussetzen, daß ihnen nicht nur ihre Publikation im Journal, sondern auch der Name ihres Verfassers eine weite Verbreitung sicherte.

Der praktische Arzt konnte aus diesen Bemühungen freilich erst dann seinen vollen Nutzen ziehen, wenn ihre Ergebnisse in die allgemeine therapeutische Lehre eingeordnet waren. Das Enchiridion medicum, das 1836 in Hufelands Todesjahr erschien, dürfte als «Vermächtnis einer funfzigjährigen Erfahrung» am eindeutigsten Antwort auf die Frage geben, ob er auch diesem Bedürfnis seiner ärztlichen Zeitgenossen gerecht geworden ist ${ }^{79}$. Denn wenn auch sein «System der practischen Heilkunde» bereits im Rahmen seiner theoretischen Lehre auf bestimmte Bäderklassen verwiesen hatte ${ }^{80}$, so nimmt doch die eigentliche Medikation der einzelnen Krankheiten im Enchiridion einen unvergleichlich breiteren Raum ein und dürfte am Ende seines Lebens die sicherste Auskunft geben, ob die Balneotherapie tatsächlich zum integrierenden Bestandteil seiner Heilmittellehre geworden ist.

Diese Therapie bleibt auch im Enchiridion von einer rationellen Empirie bestimmt, deren theoretische Grundlagen Hufeland ausführlich im System der practischen Heilkunde erläutert hatte ${ }^{81}$. Im einleitenden Abschnitt des Enchiridion wiederholt er sie nochmals ausdrücklich in den zusammenfassenden Sätzen: «Die Auswahl (Heuristik) der Heilmittel geschieht: Entweder rationell, d.h. durch eine deutliche Erkenntniß des Bedürfnisses der kranken Natur und der sich darauf beziehenden Wirkung des Heilmittels, ...; Oder empirisch, d.h. durch die erfahrungsmäßige Kenntniß der besonderen Beziehung und Heilwirkung eines Mittels auf ein bestimmtes Organ oder einen bestimmten Krankheitszustand des Organismus, .... Dahin gehören die Specifica». Diesem Postulat geht außerdem die lapidare Feststellung voraus: «Alles, was auf den menschlichen Organismus wirkt, kann als Heilmittel benutzt werden. ${ }^{82}$ Das Enchiridion erhebt daher den Anspruch, sämtliche aus der Erfahrung gewonnenen Heilmittel zu berücksichtigen. 
Die konventionelle Untergliederung des Werkes in Krankheitsklassen dürfte dabei von vornherein den Ausschluß jener Krankheitsformen gestatten, die nach Hufelands Lehre eine absolute Gegenanzeige gegen balneologische Kuren darstellen ${ }^{83}$. In diesem Sinne dürfte jedenfalls der Befund zu deuten sein, daß sich in der ersten und zweiten Klasse über die hitzigen und die intermittierenden Fieber keinerlei balneologische Verordnungen finden ${ }^{84}$. Auch die «Gastrosen » mit ihren fieberhaften Erscheinungen sind daher ausgespart, und entsprechend zurückhaltend lauten die Empfehlungen bei den Entzündungen und Blutkongestionen ${ }^{85}$. Auch hier bleiben fast sämtliche ernsthaften Entzündungen von balneologischen Maßnahmen ausgenommen, lediglich bei «Angina chronica» auf der Grundlage «hämorrhoidalischer Kongestion» wird der Karlsbader Brunnen, bei «Pleuritis chronica» das Trinken von Selterswasser mit Milch während der Nachkur und bei eitriger Nephritis und Cystitis der Gebrauch von Spaa- oder Seltersbrunnen empfohlen ${ }^{86}$. Desgleichen enthält die Klasse der «Hydropes und Pneumatosen » mit ihren Krankheitsbildern der Wassersucht und der Luftansammlungen keinerlei Hinweis auf balneologische Verordnungen, und auch in der Schwangerschaft wird von solchen Heilmaßnahmen abgesehen ${ }^{87}$.

Bei den übrigen Krankheitsklassen dagegen findet sich die ganze Vielfalt der balneologischen Indikationen aus seiner Bäderlehre: Allein in der vierten Krankheitsgruppe der «Rheumatosen» werden Patienten mit einer konstitutionellen Anlage zum chronischen Rheumatismus auf das Seebad verwiesen, bei Ischialgie und Lumbago Kuren in Wiesbaden, Teplitz oder Aachen empfohlen und bei rheumatischen Schleimhautaffektionen der Gebrauch des Egerbrunnens oder das Trinken von Selterswasser mit Milch befürwortet ${ }^{88}$. Auch in der Klasse der «Neuroses» decken sich Hufelands Verordnungen mit denen seiner balneologischen Schriften, auch hier unterscheidet er genau zwischen den verschiedenen Formen der Hypochondrie und rät bei der hypochondrischen Verstimmung auf Grund von Verstopfungen der Visceralorgane zu Karlsbad und Marienbad, während er für die neurasthenische Form die Stahlwässer und das Seebad empfiehlt ${ }^{89}$. Innerhalb der «krampfhaften Krankheiten» wiederholt sich bei Epilepsie und Chorea der Hinweis auf das Seebad, beim Tremor auf die Stahlbäder und beim "Asthma abdominale» auf Karlsbad und Ems, während er für die Cephalalgia auch hier die See und die Stahlbäder zur Wahl stellt ${ }^{90}$. Desgleichen unterscheidet er wieder zwischen den Heilanzeigen für die syphilitische, die nervöse und die viscerale Darmkolik: Bei der ersten Form empfiehlt er die Schwefelbäder, bei der zweiten das Pyrmonter Wasser und 
bei der dritten, der visceralen Form, rät er zu Karlsbad ${ }^{91}$. Unter den paralytischen Krankheiten schließ́lich verweist er die örtlichen Lähmungen auf die heißen Thermen und die neurasthenischen Magen- und Potenzstörungen abermals auf das Stahlbad Pyrmont ${ }^{92}$.

Die kursorische Überprüfung des Werkes bietet auch in den übrigen Krankheitsklassen denselben Befund: Unter den Auszehrungen (Emaciationes) lobt er wieder die prophylaktische Kur bei der Phthisis und rühmt die Wirkung von Salzbrunn, Ems und Eger bei «Phthisis purulenta» und die des Seebades bei der «Phthisis pituitosa ».93 Auch bei den «Profluvia», bei den «Suppressiones» und den Hautkrankheiten greift er in geeigneten Fällen auf balneologische Maßnahmen zurück ${ }^{94}$. Besonders häufig ist die Bädertherapie noch einmal in der zwölften Krankheitsklasse der «Dyscrasien» vertreten, wo nicht nur bei der Chlorose und Anämie Pyrmont, Driburg, Schwalbach und Kudowa, sondern auch bei der Gelbsucht mit ungehinderter Gallensekretion die Stahlwässer empfohlen werden, während der «Icterus niger» beim Steinverschluß und bei der unheilbaren Leberdesorganisation - mit seinem höchsten Grad von Gallenhemmung - Karlsbad anheimgegeben wird ${ }^{95}$. Die Gichtkranken werden schließlich nach Wiesbaden und Teplitz und zur Radikalkur auf sämtliche Schwefel- und Thermalbäder verwiesen, indes die Mercurialvergiftung nach antisyphilitischer Behandlung in Nenndorf, Weilbach, Warmbrunn und Landeck ausgeheilt werden soll ${ }^{96}$. Auch in dem Abschnitt über die Frauenkrankheiten am Ende des Werkes finden sich bei Dysmenorrhoe, Fluor albus und Sterilität weitgehend dieselben balneologischen Maßnahmen, die bereits die Bäderlehre empfohlen hatte ${ }^{97}$.

Das Enchiridion medicum zeigt damit deutlich, wie weitgehend Hufelands balneologische Erfahrungen Eingang in seine spezielle Therapie gefunden haben und mit den diätetischen und pharmazeutischen Verordnungen zu einer einheitlichen ärztlichen Praxis verschmolzen sind. Seine Verordnungen decken sich bis ins Detail mit denen seiner balneologischen Schriften, und mag auch das Werk nicht alle Badeorte erwähnen, die Hufeland in seinem Buch über die Heilquellen abgehandelt hatte, so finden sich doch fast sämtliche Heilanzeigen genannt und mit ihnen die wichtigsten Quellen der zuständigen Bäderklasse ${ }^{98}$. Dieser Befund dürfte zugleich als einhelliges Zeugnis dafür dienen, daß Hufeland nicht nur der Bäderheilkunde neue Impulse geben, sondern sie auch zum festen Bestandteil einer praktisch-empirischen Heilkunde machen wollte. Bei seinen Bemühungen, im Rahmen einer rationell-empirischen Therapie die Kenntnis differenter Pharmaka 
ständig zu erweitern, versuchte er auch die Mineralquellen in diese «Specifica » einzubeziehen ${ }^{99}$. Noch war die Frage nach der pharmakologischen Wirkung im modernen physiologischen Sinn nicht gestellt, und so standen sich Arzneistoff und Mineralquell bei Hufeland sehr viel näher als heute; denn nach seinem Verständnis dienten beide lediglich der Aufgabe, den «inneren Heilungsprozeß der Natur» anzuregen ${ }^{100}$. Nach seinem eigenen Verständnis bildete daher sein Bäderbuch einen Wegweiser in die Zukunft, und erst das Enchiridion zeigt, wie konsequent er an dieser Intention festzuhalten versuchte.

Wie in den philosophischen Grundgedanken seiner "Makrobiotik», so war Hufeland auch in der allgemeinen Badehygiene und Hydrotherapie noch immer von Francis Bacon inspiriert ${ }^{101}$. Für seine Auffassung von der praktischen Bäderlehre dürfte jedoch der Einfluß Friedrich Hoffmanns wesentlich schwerer wiegen: Schon Hufeland selbst hatte dessen Bedeutung dankbar anerkannt, und auch Emil Osann teilte zwischen beiden das Verdienst um die Begründung einer rationell-empirischen Balneologie in Deutschland ${ }^{102}$. Tatsächlich beschränkt sich dieser Einfluß keineswegs auf so grundsätzliche Forderungen wie die Beschreibung der Bäderwirkung aus eigener Anschauung oder die Bekanntmachung neu entdeckter Heilquellen. Hoffmann hat nicht nur die Mineralwasser nach chemisch-physikalischen Gesichtspunkten geordnet, um die allgemeine Wirkung der verschiedenen Bäderklassen festzustellen, er versuchte auch schon die feineren Unterschiede zwischen den einzelnen Brunnen derselben Klasse aufzuklären ${ }^{103}$. Auch ihn hatte die Erfahrung gelehrt, daß einige Mineralquellen bei sonst unheilbaren und hoffnungslosen Leiden allen übrigen Medikamenten überlegen seien, und so in seiner Ansicht bestärkt, die überaus große Feinheit, mit der die festen Bestandteile in den aquae minerales gelöst seien, erleichtere deren Aufnahme durch die Oberfläche des menschlichen Körpers ${ }^{104}$. Wie Hufeland war er auch davon überzeugt, bei Wassern, wie Pyrmont, Schwalbach und Eger, gehe mit der Verflüchtigung ihrer «spirituescentia» auch ein Teil ihrer Wirkung verloren, und wie jener bestand er daher auf dem Satz, nur der an der Quelle getrunkene Brunnen könne seine volle Heilkraft entfalten ${ }^{105}$. Selbst dem «connubium», der Mischung von Milch und kohlensauren Wassern bei Pleuritis oder Phthisis - einer Applikationsart, die in ähnlicher Form übrigens schon die antike Medizin gekannt hatte widmete Hoffmann ein eigenes Opusculum ${ }^{106}$, und auch einer genau abgestimmten Brunnendiät hat er kaum weniger Gewicht beigemessen als Hufeland $^{107}$. Gerade der Vergleich zwischen den Schriften dieser beiden Ärzte 
dürfte deutlich machen, in wie hohem Grade die Balneologie des beginnenden 19. Jahrhunderts noch immer von der Praxis der vorangehenden Zeitalter abhängig war ${ }^{108}$. Zweifellos hatte Hufelands Zeit der Bäderheilkunde mit der Zusammenfassung von See- und Mineralbad und der Entwicklung neuartiger innerer und äußerer Anwendungsformen zusätzliche Behandlungsmöglichkeiten erschlossen, auch war sie in der Bekanntmachung neuer Quellen und der empirischen Aufklärung ihrer "pharmakodynamischen» Wirkung - wie Emil Osann noch schreibt - deutlich über Hoffmann hinausgelangt, aber die Grundsätze bei der Anwendung balneologischer Maßnahmen hatten sich seit dem 17. und 18. Jahrhundert nur wenig geändert.

\section{Vitalismus und Balneologie}

Auch in Hufelands theoretischer Lehre begegnen freilich spekulative Ideen, die bereits Friedrich Hoffmann vertreten hatte: Schon jener glaubte an die eigene "virtus» einer jeden Heilquelle und an einen «mineralis spiritus», der ihre «unversehrte und wohlbehaltene Mischung und Zusammensetzung bewahren und ihre Verderbnis verhindern soll». ${ }^{109}$ Auch er billigte ihnen "genuinas et proprias vires» $\mathrm{zu}$, und selbst das «innere Laboratorium », dem bei Hufeland die Mineralwasser entspringen, dürfte sich von der «subterranea officina» Hoffmanns kaum allzu sehr unterscheiden ${ }^{110}$. Die Beispiele für solche Gemeinsamkeiten ließen sich leicht vermehren, und trotzdem mangelt dem «Scrutinium physico-medicum principiorum et virtutum in praecipuis medicatis Germaniae fontibus ", ${ }^{111}$ Hoffmanns zusammenfassendem Werk über die Heilquellen, eine so ausführliche theoretische Einführung wie Hufelands Bäderbuch, und tatsächlich ist dieser in seinen spekulativen Lehren deutlich über Hoffmann hinausgegangen.

Hier aber stellt sich die Frage, ob Hufeland in der Balneologie den romantischen Ideen seiner Zeitgenossen stärker zugeneigt war als in seinen übrigen theoretischen Lehren ${ }^{112}$. Immerhin hatte er der empirischen Heilkunde insgesamt niemals das Recht auf Spekulationen verweigert, sofern diese nur von der Erfahrung ausgingen. Lediglich die «transcendentelle» Wirklichkeitsferne rein spekulativer Lehren hat er in ihre Schranken gewiesen und als «Poesie der Medicin» abgetan ${ }^{113}$. Eine «eigene Theorie der Praxis» dagegen, die ihre Prinzipien aus der Natur nimmt und der « ein Gegebenes, kein Gedachtes » zugrundeliegt, hat er ausdrücklich befürwortet ${ }^{114}$. Seine Lehre von der Lebenskraft zeigt, daß auch «sein » System der praktischen Heilkunde nicht ohne eine solche Grundlage auszukommen vermochte, und 
schon Diepgen hat auf deren enge Anlehnung an die vitalistischen Vorstellungen seines Lehrers Blumenbach hingewiesen ${ }^{115}$. Der grundlegende Unterschied, den diese Lehre zwischen dem toten und dem lebendigen Sein wahrte, darf freilich nicht darüber hinwegtäuschen, daß Hufeland auch der anorganischen Materie Lebenskräfte zuschrieb, die an ihrem Werden und am Wechsel ihrer Formen und Strukturen einen entscheidenden Anteil nahmen ${ }^{116}$. Damit aber scheint in der Tat jener Punkt angesprochen zu sein, an dem er unter dem Einfluß der romantischen Ideen weiter ging als seine Göttinger Lehrer.

Es ist in diesem Zusammenhang nicht möglich, auf die Einzelheiten seiner Theorie von der Lebenskraft einzugehen, gerade deshalb erscheint aber ein Hinweis auf den fast unmerklichen Wandel in seiner Definition und Untergliederung des Begriffes um so notwendiger: Denn er berechtigt zu der Annahme, Hufelands Auffassung von der vis vitalis habe im Laufe der Zeit eine gewisse Akzentverschiebung zugunsten der Erregbarkeit erfahren, wenngleich er ihre umfassende Konzeption auch in späteren Jahren verteidigt und aufrechterhalten hat ${ }^{117}$. Zweifellos wird man auch in dieser allmählichen Verlagerung der Gewichte zunächst einen Tribut an den Geist der Zeit zu erblicken haben, doch man darf darüber nicht vergessen, daß seine Vorstellungen von der Erregbarkeit ursprünglich von den elektrogalvanischen Experimenten seiner Göttinger und Weimarer Zeit ihren Ausgang nahmen ${ }^{118}$. Auf ihren Erfahrungen glaubte er nach eigenem Verständnis auch dann noch zu fußen, als er unter dem Druck der Zeitströmungen die Zuständigkeit der Erregbarkeit auf organische Vorgänge ausdehnte, die er anfangs anderen «Modificationen» der Lebenskraft zugeordnet hatte. Das aber. mußte ihm um so leichter fallen, als auch in Göttingen Hallers experimentelle Arbeit nur noch die «wissenschaftliche Folie» für eine Irritabilitätslehre abgab, die deutlich unter dem Einfluß der Ideen von William Cullen stand ${ }^{119}$. Berücksichtigt man diese Umstände, die zugleich manche Unklarheiten und Widersprüche in seinen Thesen erhellen dürften, dann fügt sich auch seine theoretische Begründung der Balneologie nahtlos seiner vitalistischen Lehre ein. Nicht allein seine Balneologie, sondern sein gesamtes «System» war den romantischen Vorstellungen nähergerückt.

Seine eigenen Theorien von der Heilkunde sind es daher, die sich in seiner Bäderlehre wiederfinden, und vor einem solchen Hintergrund erscheint es nur konsequent, wenn er die chemische Kenntnis eines Mineralwassers von der praktischen Kenntnis seiner Wirkung auf den Organismus unterscheidet; denn nur diese erfaßt sein Verhalten auf «das Reagens des menschlichen 
Körpers», seinen Effekt «aufs Lebende und im Lebenden ». ${ }^{120}$ Trennt er doch auch in seiner allgemeinen theoretischen Lehre zwischen der chemischen Erkenntnis als dem «Verhalten des Todten zum Todten» und der medizinischen, die das Verhalten des Lebendigen - oder doch nur «Todtscheinenden »- zum Lebendigen wiedergibt ${ }^{121}$. Deshalb lassen sich auch die chemisch-physikalischen Prozesse der anorganischen Welt keinesfalls mit den Vorgängen im Inneren eines Organismus gleichsetzen, denn sie werden hier unter dem Einfluß der Lebenskraft in chemisch-animalische Prozesse umgewandelt ${ }^{122}$. Selbst die Trinkkur stellt daher einen chemischanimalischen Prozeß dar, und Hufeland hat in diesem Zusammenhang sogar von der "Verdaulichkeit» eines Brunnens gesprochen ${ }^{123}$. Doch auch mit diesem Begriff überträgt er nur seine allgemeine Auffassung von der Assimilation der Pharmaka im Magen- und Darmkanal auf den speziellen Bereich der Brunnentherapie, denn im nämlichen Sinn hatte er früher schon die «Verdaulichkeit und Unverdaulichkeit der Arzneymittel» erwähnt ${ }^{124}$. Diese - wie die Wirkung der Pharmaka überhaupt - wird aber nicht allein von der Art des Medikaments bestimmt, sondern auch von der Antwort des menschlichen Organismus, von der «inneren Veränderung im Lebenden», die in ihren Erscheinungsformen und in ihrer Dynamik wandelbar und keineswegs konstant ist. Der Erfolg einer Therapie hängt damit gleichzeitig von der «Reaktion des lebenden Körpers» ab, und «das einzige absolute bey der Wirkung eines Heilmittels ist seine innere Qualität und Kraft ». ${ }^{125}$ Es dürfte daher nur folgerichtig im Sinne seiner Lehre sein, wenn er über die Heilquellen schreibt: «Der chemisch und physisch vorherrschende Bestandtheil ist keineswegs auch immer der medizinisch vorherrschende; denn jener wird durch die überwiegende Quantität bestimmt, dieser durch die den Organismus vorzüglich ergreifende Qualität. ${ }^{126}$

Trotz dieser deutlichen Trennung zwischen belebter und unbelebter Welt hat aber Hufeland auch den anorganischen Stoffen bestimmte "vitale» Naturkräfte zugeschrieben, und der Satz aus seiner Bäderlehre: «Nicht blos die organischen Körper, sondern auch die unorganischen haben ihr lebendiges Seyn ", ${ }^{127}$ bedarf einer näheren Erklärung. Nach seinem Verständnis bildet die Welt des Lebens lediglich ein «höher potentiiertes Seyn der Natur selbst», oder wie er es in seinen Aphorismen sagt, « ein Erhobenseyn der Dinge zu einer höheren Stufe des Seyns ». ${ }^{128}$ Ein solches Erhobensein aber hat er auch den Heilquellen beigemessen, und selbst das gewöhnliche Wasser hält er für den Träger eines «pabulum vitae», das der chemischphysikalischen Erkenntnis unzugänglich bleibt ${ }^{129}$. 
Zweifellos mißt er dieser «Lebensnahrung» des Wassers schlechthin auch bei den Brunnenkuren eine gewisse Bedeutung bei ${ }^{130}$, doch enthalten die Mineralquellen außerdem zusätzliche Kräfte, die ihnen erst ihre spezifische Wirkung verleihen. So hat sich dem Thermalwasser die «Lebenswärme der Erde» mitgeteilt, es ist von ihr "gleichsam lebendig durchdrungen » ${ }^{131}$, und selbst beim Karlsbader Sprudel mit seinem reichen Gehalt an Mineralalkali ist dessen pharmakologische Wirkung durch die «innig beigemischte, unterirdische Wärme und manche uns noch unbekannten Stoffe» sichtbar verändert ${ }^{132}$. Mag Hufeland auch die Regel, den Brunnen früh am Morgen zu gebrauchen, von Friedrich Hoffmann übernommen haben, erst diese vitalistische Auffassung von der Kraft der Heilquellen macht eine solche Verordnung bei ihm voll verständlich; denn seine Begründung, der Quell sei in der Frühe «durch die nächtliche Ruhe stärker», erinnert unwillkürlich an sein eigenes Bekenntnis, er habe sich für seine literarischen Arbeiten stets die Morgenstunden vorbehalten, weil früh «der Geist am reinsten und produktivsten » sei ${ }^{133}$. Solche Sätze zeigen zugleich deutlich, daß er als Eklektiker auch sehr alte Vorstellungen in seine Lehre eingeschmolzen hat. Sie finden sich auch außerhalb der Balneologie und haben Pfeifer, seinen jüngsten Biographen, in anderem Zusammenhang zu der Bemerkung veranlaßt, daß er «nicht ganz frei war von Anwandlungen mystischer Ansichten der Vergangenheit $\gg{ }^{134}$

Gerade die Kraft der Thermen ohne chemischen Gehalt dient ihm daher als empirischer Beweis, daß in den Heilquellen «Imponderabilien » vorhanden sind, die ihre Wirkung hervorbringen. Bedenkt man, daß er sich mit dieser Ansicht auf einen Chemiker, wie Berzelius, berufen konnte, dann liegt es nahe, diese Imponderabilien bei ihm als vitale chemisch-physikalische Unwägbarkeiten aufzufassen ${ }^{135}$. Er fordert daher ihre Aufklärung durch eine «höhere Physik» ${ }^{136}$, in deren Zuständigkeit er bezeichnenderweise auch die Erforschung der organischen Lebensvorgänge verwiesen hatte. Diese «Physik des Lebens » mit ihrem Ziel, das Wesen der Lebenskraft zu ergründen, ist von der gewöhnlichen allerdings grundsätzlich verschieden, sie verlangt ein "fortdauerndes Experimentieren in der Region des Lebens», und nur «durch die mühsamsten Versuche wurden die einzelnen Erscheinungen dieser unbekannten Kraft unter Gesetze gebracht ». ${ }^{137}$

Für seine Auffassung vom Wesen der Heilquellen mag es dabei interessant sein, daß er gerade in diesem Zusammenhang jene unbekannte vis vitalis nicht streng auf den lebenden Organismus beschränkt, sondern auch auf Stoffe überträgt, deren Wirkung sich ohne Leben nicht bemerkbar ma- 
chen könnte. Durch solche mühsamen Versuche ließen sich nämlich nicht nur die «verschiedenen Äußerungsarten» der vitalen Grundkraft klassifizieren, «auf eben diese Weise entstanden neue Stoffe, die auch nirgends in der Natur gefunden wurden, eben weil sie blos in der Sphäre des Lebens existiren, und ihr Daseyn blos durch lebenden Organismus (durch das Reagens des Lebens) dargestellt werden kann. Zum Beweis mag der narkotische Stoff ... dienen. » ${ }^{138}$ Noch einmal läßt sich hier eine direkte geistige Brücke zwischen seiner physiologischen Theorie und seiner Bäderlehre schlagen, welche die engen Beziehungen zwischen den Heilquellen und den vitalen Wirkstoffen aufdeckt: «Denn die Mineralwasser sind darin ganz dem Weine und andern gährenden Getränken, so wie auch den narcotischen Mitteln gleich, daß sie bei offener Haut und freier Ausdünstung weniger das Sensorium angreifen, da hingegen eine durch Abkühlung bewirkte Verschließung der Haut sogleich den Zustand der Berauschung hervorbringen kann. ${ }^{139}$ Erst Hufelands Vitalismus gibt selbst solchen, scheinbar rein empirisch gemeinten Feststellungen ihren vollen Sinn.

Ganz ähnlich gelangt er durch seine Erfahrungen mit den Thermen auch zu einer Erweiterung seines Wärmebegriffs und unterscheidet die lebendige von der toten Wärme: Während aber die letzte lediglich «durch rein chemische Zersetzung hervorgebracht» wird, versteht er unter der lebendigen neben der animalischen auch die Sonnenwärme und die Erdwärme ${ }^{140}$. Diese galt ihm als vulkanisches Produkt des inneren Erdenlebens, dieses « ewigen und unbekannten, aber gewiß existierenden Prozesses», dem er daneben auch galvanische und chemische Kräfte zuschrieb ${ }^{141}$.

Es ist daher kaum verwunderlich, wenn er am Teplitzer Brunnen die «Vulkanisch-electrische Kraft» als die wirksamste rühmte, die mehr aus der Physik als aus der Chemie erklärt werden sollte ${ }^{142}$, oder wenn er den Wellenschlag des Meeres und «die dadurch hervorgebrachten electrischen und magnetischen Strömungen» für einen wesentlichen Teil der Seebäderwirkung hielt ${ }^{143}$. Hufeland hatte in seinen Göttinger Jahren unter Lichtenberg für seine Doktorarbeit elektrische Experimente an Tieren vorgenommen und versucht, «scheintote» Tiere durch galvanische Reizung wieder zum Leben zu erwecken ${ }^{144}$. In seinen Ideen zur Pathogenie hatte er dann bereits wie bei der Wärme zwischen physico-chemischer und animalischer Elektrizität unterschieden ${ }^{145}$ und außerdem von der galvanischen Reizung behauptet, sie erwecke die «Nervenkraft », die gemäß seiner Lehre wiederum eine Modifikation der Lebenskraft darstellte ${ }^{146}$. Später hat sein Schüler Bischoff in einer experimentellen Arbeit, die er unter der Anleitung seines Lehrers an 
der Charité durchführte, die galvanische Reizung als « eine schon näher mit der organischen Natur und dem Lebensprocesse verwandte ...» bezeichnet ${ }^{147}$, und am Ende seines Lebens hatte Hufeland selbst den Galvanismus sogar einen lebensvermittelnden Stoff genannt ${ }^{148}$. In diese allmähliche Aufwertung der Elektrizität im Rahmen seiner vitalistischen Lehre wird man auch seine Vorstellungen von der elektrischen Wirkung der Heilquellen einzuordnen haben. Sie geht offensichtlich mit jener bereits erwähnten Akzentverschiebung zugunsten der Erregbarkeit einher und deutet, zumindest was den Galvanismus betrifft, ebenfalls zeitbedingte Einflüsse an.

So könnten Hufelands balneologische Theorien am Ende den Eindruck erwecken, er habe der chemischen Wirkung der Quellen nur eine geringe Bedeutung beigemessen. Allein, die Tatsache, daß er als Gegner kombinierter Brunnen-Arzneimittel-Kuren die Gabe chemisch gleichartiger Medikamente gestattete ${ }^{149}$, dürfte zeigen, daß ein solches Urteil einer Einschränkung bedarf. Seine Ausführungen über Karlsbad oder die Seebäder machen deutlich, daß er die Beteiligung anorganischer Stoffe an ihrer Wirkung auf den kranken Organismus keineswegs gänzlich geleugnet hat ${ }^{150}$. Andererseits bestätigten ihm seine empirischen Beobachtungen, daß der Erfolg einer Badekur damit allein nicht erklärt war. So findet sich nicht selten der Hinweis auf geistige, gasartige oder andere flücht ge Bestandteile, «welche die Chemie noch nicht entdeckt hat, und auch nicht entdecken kann, da sie nicht in das Gebiet der chemischen Sinnlichkeit (Reagens) gehören. »151

Gerade deshalb verdient die Tatsache ein besonderes Interesse, daß er bei der Anämie die Stahlbäder verordnete, «um den chemischen Defekt im Blute zu ersetzen ", ${ }^{152}$ und es stellt sich die Frage, ob er auch diesen Vorgang vitalistischen Gesichtspunkten unterordnete. Jedenfalls hat er der vulkanischen Tätigkeit im Erdinneren nicht nur Wärmevorgänge und elektrische Strömungen, sondern auch vitale chemische Prozesse zugundegelegt ${ }^{153}$. In diesem Sinne dürften daher auch seine Worte über die Kräfte des Eisens im Pyrmonter Heilquell zu deuten sein, wenn er schreibt: «Diese Wirkung thut es (das Eisen) nun in dieser höchst verfeinerten und verflüchtigten Form des Pyrmonter Wassers in unendlich höherm Grade, und es kann nicht oft genug erinnert werden, daß die Kraft dieses und ähnlicher Mittel nicht nach dem Gewichte bestimmt werden kann, und daß ein Gran Eisen in der höchst aufgelösten verfeinerten Form eines solchen Mineralwassers kräftiger in den Organismus einwirkt, als ganze Lothe in Substanz genommen.» ${ }^{154}$ Bestimmte chemische Vorgänge werden damit gleichfalls zu einem Geschehen der lebenden Natur. 
So schließt sich der Kreis seiner Theorie und führt zu dem Kernsatz seiner Bäderlehre zurück, nach dem eine Brunnenkur den «inneren Heilungsprozeß » im Organismus in Gang bringt ${ }^{155}$. Vitale Stoffe regen damit ein vitales Geschehen an, und es kann kein Zweifel bestehen, daß in eine solche Lehre romantische Vorstellungen verwoben sind. Dennoch dürfte die Gegenüberstellung mit seinen übrigen theoretischen Schriften hinreichend gezeigt haben, daß diese Lehre einen integrierenden Teil seines allgemeinen medizinischen «Systems» bildet. Wie immer man daher seine balneologische Theorie beurteilen mag, sie läßt sich nicht von seiner allgemeinen Theorienlehre trennen und fügt sich ihr nahtlos ein. Wer beide isoliert betrachtet, dürfte eher dazu neigen, die eine wie die andere in eine engere Nachbarschaft zur Romantik zu rücken, als jener, der sie in Verbindung mit Hufelands Praxis sieht. Seine Schriften belegen jedoch, daß ihm eine so einseitige Betrachtungsweise kaum gerecht würde; denn als Praktiker im Sinne Neuburgers ${ }^{156}$ ist er auch mit seinen balneologischen Theorien nicht die breite Straße seiner Zeit gegangen, hat er sich freigehalten vom Systemzwang und von einseitigen Klassifikationen. Seine Vorstellungen sollten auch in der Bäderheilkunde als rationale Begründung für die empirische Praxis dienen und keinesfalls über diese bestimmen. Kurz, seine vis vitalis blieb auch hier das große algebraische $x$ im Ablauf der organischen Erscheinungen ${ }^{157}$ und zeigt uns besonders deutlich, wie stark auch der nüchterne Praktiker jener Epoche dem spekulativen Denken zugeneigt und wie sehr noch ein Gegner des Brownianismus und der übrigen Systeme von ihren Ansichten beeinflußt war.

\section{$\operatorname{Schlu} \beta$}

Überblickt man Hufelands Beitrag zur Bäderheilkunde noch einmal aus dem Abstand von reichlich anderthalb Jahrhunderten, dann wird man zwischen seiner Wirkung auf die eigene Zeit und auf die zweite Hälfte des 19. Jahrhunderts zu unterscheiden haben.

Zweifellos war auch der Balneologe Hufeland als Praktiker bedeutender denn als Theoretiker: Der Eklektizismus seiner Lehren vermochte auch auf diesem Gebiet keine neuen Horizonte mehr zu öffnen. Das beste an ihm war, daß er überall dort, wo Krankenbehandlung und Spekulation in Widerstreit gerieten, der praktischen Erfahrung den Vorrang erteilte, und sicherlich hat er zu seiner eigenen Zeit damit Schlimmeres verhütet. Aber auch in der Praxis konnte, wie wir heute wissen, seinem Versuch, ein Mineralwasser 
in seinem Effekt wie ein spezifisches Pharmakon abzugrenzen, kein Erfolg beschieden sein. Dennoch vertiefte er mit seiner Arbeit die praktisch-empirische Kenntnis der Anwendungsmöglichkeiten und gab so der Balneologie eine Grundlage, auf der sie in der Folge bestehen konnte, als das neue Zeitalter ihre Bedeutung immer stärker einzuengen drohte.

Die Dignität solcher ärztlichen Erfahrung bewahrte sie zugleich davor, jenen romantischen Spekulationen gänzlich anheimzufallen, die gerade in der Balneologie von Männern wie dem Dichterarzt Justinus Kerner ausgingen. Doch wenn auch Hufeland nicht wie jener die guten Geister im Wasser singen hörte, so mußten doch der aufstrebenden naturwissenschaftlichen Medizin um die Jahrhundertmitte auch seine Theorien sehr bald unannehmbar werden. Allzu eng waren in seinen Schriften Theorie und Praxis miteinander verbunden, allzu schwer wog auch der alte Ballast, den sie noch immer weitertrugen, als daß sie der neuen Zeit Genüge leisten konnten. So wurden sie beiseitegeschoben wie seine übrigen wissenschaftlichen Werke. Bereits um 1860 schrieb daher Lersch über die balneologische Literatur jener Epoche: «Fast alle diese Schriften haben für uns nichts Anziehendes mehr. ... die Balneotherapie ist in Ausdrücke gekleidet, die in unsere nüchterne Pathologie nicht mehr passen.» Und er beschließt seine Ausführungen mit dem Satz von Vetter: «Die fortlaufende Zeit überläßt uns so viel Ballast des Lebens, daß man dazu kommen muß, ihn auszuwerfen, wenn man nicht untergehen will. ${ }^{158}$

Alles in allem mögen dies die Gründe sein, warum die balneologischen Praktiker Hufeland auf lange Zeit eine dankbare Erinnerung bewahrten, sein wissenschaftliches Werk aber so rasch in Vergessenheit geriet. 


\section{Wissenschaftliche Anmerkungen}

Hufelands Journal, von 1795 bis 1808 (Bände 1-27) unter dem Titel: Journal der praktischen Arzneikunde und Wundarzneikunsı und ab 1808 (Band 28) unter dem Titel: Journal für praktische Heilkunde erschienen, wird unter dem Sigle: J.H. zitiert.

1. F. von Oefele (Geschichte der Balneologie und der Grenzgebiete in der Neuzeit, in: Puschmann, Neuburger, Pagel, Handbuch der Geschichte der Medizin, Band II, Jena 1903, S.597) erwähnt Hufeland überhaupt nur als Schwiegervater von Emil Osann, dessen Handbuch er freilich nennt (E. Osann, Physikalisch-medicinische Darstellung der bekannten Heilquellen der vorzüglichsten Länder Europa's, Band I, 2. Auflage, Berlin 1839; Band II, 2. Auflage, Berlin 1841; Band III, 1 und 2 postum herausgegeben von F.ZABEL, Berlin 1843). - A. MARTIN wiederum zitiert ihn zwar hin und wieder zu einzelnen Fragen, doch einen zusammenfassenden und generellen Hinweis auf seine Leistungen bleibt er schuldig (Deutsches Badewesen in vergangenen Tagen, Jena 1906, S.136, 218, 268, 366 und 402; Abriß der Balneologiegeschichte, in: Dietrich UND KaMiner, Handbuch der Balneologie, medizinischen Klimatologie und Balneographie, Band I, Leipzig 1916, S.38).

2. Сн. W.Hufeland, Nöthige Erinnerung an die Bäder und ihre Wiedereinführung in Deutschland, nebst einer Beschreibung der russischen Dampfbäder, Journal des Luxus und der Moden 5 (1790) $377 \mathrm{ff}$; wieder abgedruckt in: Gemeinnützige Aufsätze zur Beförderung der Gesundheit und des Wohlseyns und vernünftiger medicinischer Aufklärung, Band I, Leipzig 1794, S. 135 ff. (im folgenden nach dieser Ausgabe zitiert).

3. Einige Schönheitsmittel nicht aus Paris, Journal des Luxus und der Moden 3 (1788) $14.2 \mathrm{ff}$; ; wieder abgedruckt in: Gemeinnützige Aufsätze etc., Band I, S.96ff.

4. Vgl. seinen Aufsatz: Über die Verlängerung des Lebens, Der Neue Teutsche Merkur vom Jahre 1792, S. 261; und insbesondere: (Makrobiotik) Die Kunst das menschliche Leben zu verlängern, Jena 1797, 1.Auflage, II.Praktischer Theil, II.Abschnitt, Kapitel 11: Reinlichkeit und Hautkultur, S.592ff. - Speziell für die Kinderhygiene siehe: Über die wesentlichen Vorzüge der Inoculation vollkommne und unvollkommne Blattern, und andere dahin gehende Punkte, desgleichen über verschiedene Kinderkrankheiten und sowohl diätetische als medizinische Behandlung der Kinder, Leipzig 1792, S. 240 ff., und später: Guther Rath an Mütter über die wichtigsten Puncte der physischen Erziehung der Kinder in den ersten Jahren, Berlin 1799, Kapitel: Kaltes Waschen, und Kapitel: Laue Bäder, S. $13 \mathrm{ff}$.

5. G. Сн.Lichtenberg, Warum hat Deutschland noch kein großes öffentliches Seebad?, ursprünglich erschienen in: Göttinger Taschenkalender 1793, S. 92 ff.; wieder abgedruckt in: Georg Christoph Lichtenbergs Vermischte Schriften, neue, vermehrte von dessen Söhnen veranstaltete Originalausgabe, Band 6, Göttingen 1845, S.11 ff., und neuerdings in: G.C.Lichtenberg, Tag und Dämmerung (Sammlung Dieterich, Band 75), Leipzig 1942, S. $445 \mathrm{ff}$ - - CH. W. Hufeland, Seebad, Neueste Annalen der französischen Arzneykunde und Wundarzneykunst 2 (1793) 302. - W. BREDNow verweist in seiner lesenswerten Studie: Christoph Wilhelm Hufeland, Arzt und Erzieher im Lichte der 
Aufklärung (Sitz.-Ber. d. Sächs.Akad. d. Wiss. zu Leipzig, Math.-nat. Kl., Band 105, Heft 6, Berlin 1964, 2. Auflage, S. 10) mit Recht darauf, daß sich Lichtenberg in seinem Aufruf auf HufeLands Nöthige Erinnerung an die Bäder und ihre Wiedereinführung in Deutschland bezieht, doch andererseits hat Lichtenberg in seinem Aufsatz als erster die Gründung von Seebädern in Deutschland gefordert, während Hufeland das Schwimmen im Meer zunächst nur nebenher gemeinsam mit dem Baden in Bächen und Flüssen ganz kurz erwähnt hatte (S.155; vgl zu dieser Frage J.D. W.SACHSE, Medicinische Beobachtungen und Bemerkungen, Band I: Über Bäder, besonders in Beziehung auf die Seebäder bei Doberan, Berlin 1835, S.44). Er hat Lichtenbergs Anliegen freilich sofort zu dem seinen gemacht und, wie sich im folgenden zeigen läßt, wirksam gefördert. Ob sich Lehrer und Schüler über ihr Vorgehen abgesprochen hatten, läßt sich heute nicht mehr aufklären; Lichtenbergs Briefwechsel gibt jedenfalls keinerlei Anhaltspunkte (überprüft wurden folgende Editionen: A.LEItzManN und C. SchüDDEKopF, Lichtenbergs Briefe, Band 1-3, Leipzig 1901-1904; E. EBsteIn, Aus G. Ch. Lichtenbergs Correspondenz, Stuttgart 1905; M.Doмke, Ungedruckte Briefe Lichtenbergs, Berlin 1925, Privatdruck; G.Сн.Lichtenberg, Schriften und Briefe, herausgegeben von W.Promies, Band 4, Darmstadt 1967).

6. Die Kunst das menschliche Leben zu verlängern, S.594f. - Der Inhalt dieses Abschnitts dürfte übrigens bis in die Formulierung einzelner Sätze Hufelands Abhängigkeit von Lichtenberg ausweisen.

7. z.B.: Nöthige Erinnerung an die Bäder, S.152f.; vgl. auch E.BaymanN, Christoph Wilhelm Hufeland und die praktische Medizin, Düsseldorf 1964 (Diss. med.) S.14.

8. Über lauwarme Bäder (Frankfurt am Main 1802; zur Verfügung stand die Ausgabe: Berlin o. J. [um 1803]), eine Monographie, die noch einmal ausführlich das Thema vom Nutzen der Bäder für das Hautorgan aufnimmt (S.16ff.; vgl. zu dieser Frage auch A.Martin, Deutsches Badewesen, S.395) und damit inhaltlich an seine Nöthige Erinnerung an die Bäder anschließt, wo er bereits die «Unterlassung der Bäder» als «Versäumung der Hautkultur» bezeichnet hatte (S.145). - Ferner: Das Element des Wassers als Heilmittel, J.H.38 (1814) St.1, S.1ff., ein Aufsatz, in dem er sich nicht nur für Bäder, sondern auch für einfache Wassertrinkkuren einsetzt. Außerdem griff er später in den Streit um den Wert der kalten Bäder bei fieberhaften Erkrankungen ein, indem er 1821 im Journal eine Preisfrage zu diesem Thema stellte. Siehe: Anzeige über die Beantwortung der in diesem Journal 1821 aufgestellten Preisfrage «Über die äußerliche Anwendung des kalten Wassers in hitzigen Fiebern», Einleitung des Herausgebers zum Supplementstück des Journals 55 (1822), in dem die drei preisgekrönten. Arbeiten von Fröhlich, Reuss und Pitschaft publiziert wurden (als eigener Druck erschienen: Berlin 1823). Zu den geschichtlichen Zusammenhängen vgl. P.DIEPGEN, Geschichte der Medizin, Band II 1, Berlin 1959, S.38f.

9. Vgl. Praktische Übersicht der vorzüglichsten Heilquellen Teutschlands, Berlin 1815, S.1f. - Allerdings wäre es verfehlt, sein frühes Interesse etwa auf Kindheitseindrücke, d.h. auf seinen Geburtsort Langensalza oder auf Tennstädt, den Herkunftsort seiner Familie, zurückzuführen; denn die Quellen dieser beiden Badeorte waren damals noch nicht entdeckt und sind erst später in Nutzung genommen worden. Vgl. K. PfEifER, 
Christoph Wilhelm Hufeland, Mensch und Werk, Halle 1968, S.201. - Zu den balneologischen Schriften von Friedrich Hoffmann siehe: Opera omnia physico-medica, Tom. V, Genf 1740, Opuscula de aquis mineralibus earumque salutari virtute, S.131 ff.

10. Vgl. Reisebemerkungen im Herbst 1823, J.H.57 (1823) St. 5, S. 113.

11. Zur Bedeutung dieser Zeitschrift vgl. W.A.L.v.Brunv, Medizinische Zeitschriften im 19. Jahrhundert, Stuttgart 1963, S.9ff.

12. J.H.1 (1795) St.1, S.XVIf.

13. Etwas über das Limmerschwefelwasser, J.H.1 (1796) St. 3, S.416ff.

14. Flüchtige Reisebemerkungen im Sommer 1798, J.H.7 (1799) St.2, S.156ff. (wieder abgedruckt in: C. W. HufeLAND, Kleine medizinische Schriften, Band II, Berlin 1823, S. $387 \mathrm{ff}$.).

15. Ibidem S. 157 f. - Zu Sagard vgl. M.v. WILLICH, Vorläufer einer künftigen ausführlichen Beschreibung des Gesundbrunnens zu Sagard auf der Insel Rügen, Stralsund 1795. Die beiden Brüder v. Willich waren die Begründer des Bades, sie werden auch von Hufeland in seinem Bericht erwähnt.

16. Zum Zeitpunkt der Erblindung vgl. Verfasser, Bemerkungen zur Datierung von Hufelands rechtsseitiger Erblindung, Gesnerus 26 (1969) $249 \mathrm{ff}$.

17. Hufeland, Leibarzt und Volkserzieher, Selbstbiographie von Christoph WiLhelm Hufeland, neu herausgegeben und eingeleitet von W.v.BrunN, Stuttgart 1937, S. 85. - Die Kunst das menschliche Leben zu verlängern, S.596. - Zu Doberan vgl. S. G.VogeL, Über den Nutzen und Gebrauch der Seebäder, nebst der Ankündigung einer öffentlichen Seebadeanstalt, welche an der Ostsee in Mecklenburg angelegt wird, Stendal 1794. Siehe auch A.MARTIN, a.a. O., S. $62 \mathrm{f}$.

18. Flüchtige Reisebemerkungen im Sommer 1798, S.166.

19. Ibidem S. $158 \mathrm{ff}$. und S. $164 \mathrm{ff}$.

20. Ibidem S.160. In seinen Reisebemerkungen übergeht er den Mißerfolg der eigenen Kuren mit Stillschweigen, und so erscheint ein gewisser Widerspruch zu den Angaben in dem Anm. 17 zitierten Abschnitt seiner Selbstbiographie unausweichlich. Auch wird man berücksichtigen müssen, daß seine Lebenserinnerungen in einem größeren zeitlichen Abstand geschrieben und ursprünglich nur für einen engen Verwandtenund Freundeskreis bestimmt waren.

21. Selbstbiographie, Ed. W.v.BrunN, S.93.

22. Practische Blicke auf die vorzüglichsten Heilquellen Teutschlands, Abschnitt Pyrmont, J.H.27 (1808) St.2, S.6. In der zusammenfassenden Buchedition unter dem Titel: Praktische Übersicht der vorzüglichsten Heilquellen Teutschlands (siehe Anm.9 und im folgenden S. 196), Berlin 1815, S. 70, findet sich nach dem Tod der Königin dieser Satz folgendermaßen abgewandelt: «..., der unsrer allverehrten, leider jetzt von uns betrauerten, Königin neues Leben, den letzten schönen Abend ihres Daseins, und Kraft gab, die nachfolgende Nacht zu ertragen.»

23. Gesundbrunnen zu Meinberg, J.H.24 (1806) St.4, S. $161 \mathrm{ff}$. 
24. Nenndorfs asphaltische Schwefelquelle, J.H.4. (1797) St.4, S. 830 ff.; vgl. L.P. SchröTER, Über die vorzüglichen Heilkräfte des Nenndorfer Schwefelwassers, Rinteln 1797. Auch in der Folgezeit nahm Hufeland Neuerscheinungen auf dem Gebiet der balneologischen Literatur zum Anlaß, in den kurzen Nachrichten und medizinischen Neuigkeiten des Journals auf Heilanzeigen und Erfolge einzelner Bäder gesondert hinzuweisen; vgl. z. B.: Wildunger Wasser und Aqua mephitica alcalica beym Stein, J.H.9 (1800) St. 4, S. $180 \mathrm{ff}$. Siehe auch die folgenden Anmerkungen.

25. Neues Seebad auf der Ostfriesischen Insel Norderney und zu Colberg, J.H.13 (1802) St. 4 , S. 180 f.

26. Über den Gesundbrunnen zu Sagard, auf der Insel Rügen (Aus einem Briefe 1799), J.H. 9 (1800) St. 2, S. 195 ff., und: Salzbäder in der Gicht, J.H. 10 (1800) St. 4, S. 179 ff. (wieder abgedruckt in: Kleine medizinische Schriften, Band III, Berlin 1825, S. $290 \mathrm{ff}$.). - Gefahren der fixen Luft und der luftsauren Mineralwasser bey Schwangerschaften, J.H.5 (1798) St.3, S.671f. - Empfehlung des Driburger Gesundbrunnens, J.H.21 (1805) St.3, S.176ff. - Der Selkenbrunnen am Harze, J.H.28 (1809) St.6, S.129f.

27. J.H.14 (1802) St.2, S.193ff. (wieder abgedruckt in: Kleine medizinische Schriften, Band III, S. $466 \mathrm{ff}$.$) .$

28. Ibidem S. 194.

29. Ibidem S.195ff. - Vgl. zu dieser Frage: Ideen über Pathogenie und Einfluß der Lebenskraft auf Entstehung und Form der Krankheiten, Jena 1795 (1.Auflage), S.64; Über die Verlängerung des Lebens, Neuer Teutscher Merkur vom Jahre 1792, S.242; siehe auch die folgenden Ausführungen auf S.211 f. und 215.

30. Ibidem S. 194.

31. Vgl. C. С. МаттнӓI, Von welchen Ursachen hängt der große Nutzen der Brunnen- und Badecuren eigentlich ab? J.H.19 (1804) St. 2, S. 5 ff.

32. Ibidem S. 59 .

33. Practische Blicke auf die vorzüglichsten Heilquellen Teutschlands: Allgemeine Bemerkungen über Mineralwasser und ihren Gebrauch, J.H.27 (1808) St.1, S.9ff.; Pyrmont, Driburg, Schwalbach, St. 2, S. 5 ff.; Carlsbad, St. 3, S. 5 ff.; Nenndorff, Eilzen, St. 4, S. 80 ff.; Töplitz, J.H. 28 (1809) St. 1, S. 6 ff.; Eger, St. 2, S. 47 ff.; Selters, Fachingen, Schwalheim, Bilin, Wildungen, St. 4, S. 1 ff.; Das Seebad, St. 5, S. 49 ff.; Beschluß, J.H. 29 (1809) St. 4, S.1ff. Buchedition: Praktische Übersicht der vorzüglichsten Heilquellen Teutschlands nach eignen Erfahrungen, Berlin 1815.

34. Ibidem S. VIf.

35. Zusätze eines Ungenannten, J.H.27 (1808) St.2, S. 33 ff., und J.H.29 (1809) St. 4, S.1ff. = Buchedition S.258ff. Die Identität dieses Ungenannten ist bis heute nicht geklärt, doch erscheint es unwahrscheinlich, daß er nur fingiert war und die Zusätze in Wirklichkeit von Hufeland selbst geschrieben sind; vgl. K. Pfeifer, a. a. O., S. 202.

36. Zum folgenden vgl. J.H.27 (1808) St.1, S.9ff. = Buchedition S.1ff. (in der Buchedition finden sich einige - meist geringfügige Zusätze). 
37. Ibidem S.17f. = Buchedition S.12f.

38. Buchedition S. VII.

39. Ibidem S. 17 = Buchedition S. 12. - Vgl. F. Hoffmann, De elementis aquarum mineralium recte dijudicandis et examinandis, § XXXVIff., a.a.0., S.137ff.

40. Ibidem S. $21 \mathrm{ff}$. = Buchedition S. $17 \mathrm{ff}$.

41. Zur Brunnendiät siehe ibidem S. $40 \mathrm{ff}$. = Buchedition S. $38 \mathrm{ff}$. Anstelle von $~ «$ Regimen der Seele» steht in der Buchedition «Diät der Seele» (S.45 = S.43).

42. Ibidem S.54ff. - Buchedition S. $54 \mathrm{ff}$.

43. Ibidem S. $60 \mathrm{ff}$. = Buchedition S. $60 \mathrm{ff}$.

44. Ibidem S. $62 \mathrm{ff}$. $=$ Buchedition S. $62 \mathrm{ff}$.

45. Ibidem St. 2, S. 7 und $29=$ Buchedition S. 71 und 90 .

46. Ibidem St. 3, S. $10=$ Buchedition S. $140 \mathrm{f}$.

47. Ibidem St. 2, S.11 ff. = Buchedition S. 75 ff., und St. 3, S. 14 ff. = Buchedition S. $145 \mathrm{ff}$.

48. J.H.28 (1809) St.5, S.51f. = Buchedition S.239.

49. Ibidem S. 53 ff. $=$ Buchedition S. $240 \mathrm{ff}$.

50. Vgl. Revision der Heilquellen, J.H.34 (1812) St.3, S.110.

51. Vgl. J.H. 46 (1818) St. 1, S. 3 ff. und die Fortsetzungen in Band 69 (1829) St. 1, S. $7 \mathrm{ff.}$, und Band 72 (1831) St.1, S. $7 \mathrm{ff}$.

52. A. DeStourdza, C.W. Hufeland esquisse de sa vie et de sa mort chrétiennes, Berlin 1837, S. 12 .

53. Praktische Übersicht der vorzüglichsten Heilquellen Teutschlands, S.48f. und 95.

54. Bitte um bessere Einrichtung und Benutzung der Heilquelle zu Selters, J. H. 45 (1817) St.5, S.119ff. - Neue Erfindung die Zersetzung der eisenhaltigen Mineralwasser bei dem Transport zu verhindern, J.H.62 (1862) St. 5, S. 136 f. und: Nöthige Aufmerksamkeit auf den jetzigen Unterschied des versendeten Egerwassers, J.H.74 (1832) St. 5, S.126f. - Verbrauch der Mineralwasser in Berlin im Jahre 1822, J.H. 58 (1824) St.1, S.114.

55. Verfälschung der Mineralwasser, J.H.27 (1808) St.4, S.166ff. - Revision der Heilquellen loc. cit.

56. Nöthige Erinnerung an die Bäder, S.157, und: Praktische Übersicht, S. 182 Anm. - Zur Einführung des Badethermometers vgl. A.MARTin, a. a.O., S. 402.

57. Flüchtige Reisebemerkungen im Sommer 1816, J.H.43 (1816) St.4, S. 129 ff.

58. Flüchtige Reisebemerkungen auf einer Reise nach Nenndorf, Eilsen, Meinberg, Pyrmont, Ems, Schwalbach, Schlangenbad, Wiesbaden, im Sommer 1821, J.H.53 (1821) St. 5, S. $124 \mathrm{ff}$.

59. Ibidem S. 133.

60. Vgl. S.199. 
61. Reisebemerkungen im Herbst 1823, S.113ff.

62. Vgl. Selbstbiographie, Ed. W.v. BrunN, S.113.

63. Reisebemerkungen im Herbst 1823, S.125f.

64. Ibidem S.115.

65. Vgl. Wunderbare Wirkung des Carlsbades auf die Erzeugung des Callus, J. H. 43 (1816) St. 4, S.135f., und Kraft der Thermen ohne chemischen Gehalt, J.H.62 (1826) St.1, S. $99 \mathrm{f}$.

66. Neue Bestätigung der großen Kraft des Aachener Bades bei syphilitischen Krankheiten, J.H.65 (1827) St.6, S.146f., und Eger's Salzbrunnen, J.H.70 (1830) St.4, S. $123 \mathrm{f}$.

67. Das neue Schwefelbad zu Berka an der Ilm, J.H.43 (1816) St.1, S.123f. und Einführung eines neuen eisenfreien Mineralwassers des Ludwigsbrunnens in der Wetterau, J.H.82 (1836) St. 4, S. $112 \mathrm{ff}$.

68. Die hepatischen Gasbäder zu Eilsen, J.H.34 (1812) St.4, S.115f. - Zu seinen Hinweisen auf die Gas- und Schlammbäder in Nenndorf und Meinberg vgl. seine bereits zitierten Aufsätze: Flüchtige Reisebemerkungen im Sommer 1816, S. 130 f. und Flüchtige Reisebemerkungen ... im Sommer 1821, S.126f.

69. Seebad zu Norderney in Ostfriesland, J.H.44 (1817) St.6, S.100f.

70. Vgl. z. B.: Badechronik vom Jahr 1821 mit Nachrichten über Franzensbad und Driburg, J.H. 55 (1822) St. 1, S. 89 ff., oder Badechronik vom Jahre 1822 mit Nachrichten über Marienbad, Cuxhaven und Rehburg, ibidem St.4, S.117ff.

71. J.H.57 (1823) St.5, S.129.

72. Der Egerbrunnen, J.H.55 (1822) St. 4, S.123 ff.

73. Vgl. A.Treumann, Über das Freienwalder Bad, Berlin 1827. - Th. Fontane, Wanderungen durch die Mark Brandenburg, neue Ausgabe herausgegeben von seinen Söhnen Theodor und Friedrich Fontane, Teil 2: Das Oderland, Berlin 1925, S.31 ff. und $47 \mathrm{ff}$.

74. Praktische Übersicht, S.133 (nicht enthalten in der Aufsatzreihe: Practische Blicke).

75. Noch einige Worte über Heilquellen - Bitte an die Ärzte, die ihre Kranken dahin schicken, J.H.63 (1826) St. 4, S.124ff.

76. Zwölf wöchentliche und dennoch glücklich beseitigte Harnverhaltung, mit darauffolgender Blennorrhoe der Blase. Großer Nutzen des Wildunger Wassers bei Blasenkrankheiten, J.H.75 (1832) St. 5, S. $17 \mathrm{ff}$.

77. Ibidem S. 23.

78. Vgl. Anm. 12 .

79. Enchiridion medicum oder Anleitung zur medizinischen Praxis, Berlin 1836. Noch im selben Jahr, wenige Wochen vor seinem Tode, ging die 2. Auflage in Druck. (Benutzt wurde die 3. Auflage, die bereits ein Jahr später erschien; nach ihr wird im folgenden zitiert.) 
80. Z.B. Band I, Jena und Leipzig 1800, S.331: Unter den Eisenmitteln der Hinweis auf Pyrmont, Driburg, Spaa, Schwalbach; oder Band II 2, Jena und Leipzig 1805, S.44: Bei der Schwefeltherapie gegen Hautkrankheiten der Hinweis auf Aachen, Nenndorf, Landeck, Limmer und Baden.

81. Ibidem Band I, S.56, 132 und $199 \mathrm{f}$.

82. Enchiridion S.73 (Sperrdruck von Hufeland).

83. Vgl. S. 200 .

84. Enchiridion S.84ff.; lediglich beim gastrischen Fieber wird das Trinken von Selterswasser anheimgestellt (S.119).

85. Zu den Gastrosen (5. Krankheitsklasse) vgl. ibidem S. 234ff. und zu den Entzündungen und Blutcongestionen (3. Krankheitsklasse) S. $150 \mathrm{ff}$.

86. Ibidem S. 180, S.194 und S.205f.

87. $\mathrm{Zu}$ den «Hydropes und Pneumatoses» (8. Krankheitsklasse) vgl. S.418ff. und zur Schwangerschaft im Abschnitt über die Frauenkrankheiten S. $693 \mathrm{ff}$.

88. Ibidem S.225, S.228f. und S.232. - Der Begriff Rheumatismus ist bei Hufeland noch im alten humoralen Sinn gebraucht; denn der Eklektizismus seines theoretischen «Systems» ließ durchaus auch humorale Vorstellungen gelten. Trotzdem darf man ihn nicht mehr als Anhänger Galens betrachten, und es ist sicherlich unzulässig, im Pergamener seinen «großen Lehrmeister » zu sehen, wie es P. LÜтH in einer seiner eigenwilligen historischen Interpretationen getan hat (Geschichte der Geriatrie, Stuttgart 1965, S. 195). Das verbietet allein schon Hufelands Aufsatz: Hippokrates und Galenus, Natur und Schule (J.H.48 [1819] St.1, S. 1 ff.).

89. Zur Untergliederung der «Neuroses» (6. Krankheitsklasse) in die Krankheistgruppen: Gemütskrankheiten, Krampfhafte Krankheiten und Paralytische Krankheiten siehe S. 251 ff., S. 272 ff. und S. 333 ff. - Zur Hypochondrie siehe S. 264 ff. und vgl. oben S. $200 \mathrm{f}$.

90. Ibidem S. 275 und 278, S. 285, S. 292, S. 317.

91. Ibidem S. $325 \mathrm{f}$.

92. Ibidem S. 355, S. 363 und S. 367.

93. Zur prophylaktischen Kur vgl. ibidem S. $387 \mathrm{ff}$. Dabei nennt er Ems und Eger für die pulmonale und zusätzlich Marienbad und Selters für die abdominale Form. Zur Phthisis purulenta vgl. S. 391 und zur Phthisis pituitosa S.406. - Zur Balneotherapie der «Tabes dorsualis » innerhalb dieser 7. Krankheitsklasse siehe außerdem S. 410.

94. Innerhalb der «Profluvia » (9. Krankheitsklasse) empfiehlt er bei den « Haemorrhoides vesicae» Wildungen und Karlsbad (S.460), bei der Haematurie Wildunger oder Selterswasser (S.466), bei der Harnruhr (Diabetes) nennt er Karlsbad (S.475). Innerhalb der «Suppressiones» (10. Krankheitsklasse) verordnet er bei der «Obstructio alvi» und bei der «Dyscopria » wiederum Karlsbad (S.504), und bei der adynamischen oder paralytischen Form der Ischuria erwähnt er das Wildunger Wasser (S.512). Unter den Hautkrankheiten (11. Krankheitsklasse) empfiehlt er Nenndorf und Eilsen für die Hautflechten (S.574). 
95. Ibidem S.596 und S.602. - Zur Wirkung der Mineralbäder bei Konkrementbildungen siehe auch die 13. Klasse der «Desorganisationes», wo gegen den Harnstein der Gebrauch des Wildunger, Geilnauer und Fachinger Brunnens empfohlen wird (S.675), und gegen den Gallenstein abermals Karlsbad (S.676).

96. Ibidem S.624 und S.627, S. 650 .

97. Ibidem S. 690 und S. 692 (Karlsbader und Saidschützer Bitterwasser); S. 714 (Schwefel-, See- und Eisenbäder); S. 717 (Ems und Pyrmont).

98. Zu Hufelands Klassifikation der Mineralquellen vgl. seine «Tabellarische Übersicht der vorzüglichsten Heilquellen Teutschlands» in: Praktische Übersicht, S. $299 \mathrm{ff}$.

99. $\mathrm{Zu}$ seinen Bemühungen um neue Arzneistoffe vergleiche man nicht nur seine Monographie Erfahrungen über die Kräfte und den Gebrauch der salzsauren Schwehrerde (!) in verschiedenen Krankheiten (Erfurt 1792; vermehrt und verbessert in der 2. Auflage, herausgegeben unter dem Titel: Vollständige Darstellung der medicinischen Kräfte und des Gebrauchs der salzsauren Schwererde, Berlin 1794), sondern vor allem seine entsprechenden Aufsätze im Journal, von denen der überwiegende Teil in den Kleinen medizinischen Schriften unter dem Titel: «Praktische Bemerkungen über einige Arzneimittel» und: «Praktische Bemerkungen über verschiedene Arzneimittel» zusammengefaßt ist (Band III, S. $260 \mathrm{ff}$. und $4.77 \mathrm{ff}$.). Dabei mag es bezeichnend sein, daß in die Reihe dieser pharmakognostischen Notizen auch sein kurzer, bereits zitierter balneologischer Aufsatz «Salzbäder in der Gicht» Aufnahme gefunden hat. - Zu seinen Bemühungen, den «spezifischen Karakter, das Auszeichnende einer jeden der vorzüglichen Heilquellen herauszuheben» vgl. Praktische Übersicht, Vorrede S. VI.

Seine Bemühungen um eine Vermehrung spezifischer Pharmaka hinderten ihn freilich nicht, gegen Auswüchse auch in diesem Bereich Stellung zu nehmen. Siehe seine Bemerkungen zu dem Aufsatz von J.G.OBenteuffer, Practische Erfahrungen, mit verschiedenen in diesem Jahrhundert, besonders der zweiten Hälfte desselben, erfundenen oder hochgepriesenen Mitteln, J.H.9 (1800) St.3, S. 77 ff. Doch auch hier finden sich die bezeichnenden Sätze: "Wahrlich, kaum ein Arzt haschte mehr nach neuen Mitteln, als ich, daher mögen vielleicht auch wenige gefunden werden, die mehr betrogen und darum mißtrauischer geworden sind» (S. 79).

100. Enchiridion S.71f.

101. Vgl. Die Kunst das menschliche Leben zu verlängern, S.XV, und: Über die Verlängerung des Lebens, S. 261.

102. Vgl. S. 192 und Anm.9, und: E.Osann, Art. Hufeland, in: Encyclopädisches Wörterbuch der medicinischen Wissenschaften, Band 17, Berlin 1838, S. 148.

103. F. Hoffmann, a. a. O., S.158f.

104. Ibidem S. 135 und 132.

105. Ibidem S.132f. und S.155. Vgl. bei HufeLAND z.B.: Praktische Übersicht, S.171 (siehe auch oben S.198).

106. F. Hoffmann, De connubio aquarum mineralium cum lacte longe saluberrimo, a.a. O., S. 222 ff.; vgl. bei Hufeland z. B.: Practische Blicke, J. H. 27 (1808) St. 2, S. 32, oder: Bitte um bessere Einrichtung der Heilquelle zu Selters, S.120. 
107. Z.B. ibidem S. 169 (§ XXVII).

108. Vgl. zu dieser Frage J.STEudeL, Therapeutische und soziologische Funktion der Mineralbäder im 19. Jahrhundert, in: Studien zur Medizingeschichte des 19. Jahrhunderts, herausgegeben von W.ArTelt und W.RüEgG, Band I: Der Arzt und der Kranke in der Gesellschaft des 19. Jahrhunderts, Stuttgart 1967, S. 83.

109. F.Hoffmann, a.a.O., S.155 (vgl. dazu bei Hufeland z.B. Praktische Übersicht, S. $58 \mathrm{f}$.$) .$

110. Ibidem S.131 (vgl. dazu bei Hufeland z. B. Revision der Heilquellen, S.110; siehe auch: Flüchtige Reisebemerkungen ... im Sommer 1821, S.131).

111. F. Hoffmann, a. a. O., S.141 ff.

112. Vgl. J.SteudeL, a. a. O., S. 83 f.

113. Über den Begriff der practischen Medicin und deren Verschiedenheit von der speculativen in Beziehung auf die Lage der Heilkunde während der medicinischen Revolutionszeit zu Ende des vorigen und Anfang des jetzigen Jahrhunderts, J.H.19 (1804) St. 1, S. XVII (nur auszugsweise abgedruckt in: Kleine medizinische Schriften, Band IV, Berlin 1828, S.4).

114. Enchiridion, Abschnitt Praxis, S.79; entnommen aus: Aphorismen eines freien Arztes, abgedruckt in: Neue Auswahl kleiner medizinischer Schriften, Band I, Berlin 1834, S.336.

115. Vgl. P.Diepgen, Christ. Wilhelm Hufeland und die Medizin seiner Zeit, Münch. Med. Wschr. 83 (1936) Nr.35, Separatum S. 3; abgedruckt in: Medizin und Kultur, Stuttgart 1938, S.211. Siehe auch A.Göschen in seiner Einleitung zu: Christian (!) Wilhelm Hufeland, eine Selbstbiographie, Deutsche Klinik 15 (1863) 122 und insbesondere in seinen erläuternden Nachträgen, S.264f.

116. Vgl. Über die Verlängerung des Lebens, S.242 und andernorts.

117. Diese Frage bedarf einer eigenen ausführlichen Untersuchung, die vor allem seine Schriften aus dem Zeitraum zwischen 1790 und 1810 zu berücksichtigen hätte. - Zu Hufelands Festhalten an seiner Gesamtkonzeption von der Lebenskraft siehe seine Vorrede zu Band III seiner Kleinen medizinischen Schriften, Berlin 1825, S. VIff.

118. Vgl. Selbstbiographie, Ed. W.v. Brunn, S.64 und 71.

119. Vgl. M. Neuburger, in: Puschmann, Neuburger, Pagel, Handbuch der Geschichte der Medizin, Band II, S. $89 \mathrm{ff}$.

120. Aufforderung an die Brunnenärzte Deutschlands, S.194.

121. Die Atmosphäre in ihren Beziehungen auf den Organismus, J.H.31 (1810) St.5, S.4.

122. Ideen über Pathogenie, S. $63 \mathrm{ff}$.

123. Praktische Übersicht, S. $17 \mathrm{ff}$.

124. System der practischen Heilkunde, Band I, S.166.

125. Ibidem S. $133 \mathrm{ff}$. und $150 \mathrm{ff}$. 
126. Praktische Übersicht, S. 300 .

127. Ibidem S.9.

128. Vgl. Die Welt des Lebens. Die höhere Ordnung der Dinge. Die höhere Physik, in: Neue Auswahl kleiner medizinischer Schriften, Band I, S.51; - Aphorismen eines freien Arztes, ibidem S. 336.

129. Das Element des Wassers als Heilmittel, J.H. 38 (1814) St.1, S. 5.

130. Ibidem S. 8 .

131. Flüchtige Reisebemerkungen ... im Sommer 1821, S.131.

132. Praktische Übersicht, S.139f.

133. Ibidem S.29 und Selbstbiographie, Ed.W.v.BrunN, S.61.

134. K.PFeifer, a. a.O., S. 103.

135. Kraft der Thermen ohne chemischen Gehalt, J.H.62 (1826) St. 1, S. 99 f.

136. Praktische Übersicht, S.137.

137. Die Welt des Lebens. Die höhere Ordnung der Dinge. Die höhere Physik, S.52f.

138. Ibidem.

139. Praktische Übersicht, S. 28.

140. Großer Werth der heißen Quellen - Verschiedenheit der unterirdischen Wärme von der gewöhnlichen, J.H.48 (1819) St.4, S.123.

141. Ibidem S.122.

142. Reisebemerkungen im Herbst 1823, S.123.

143. Praktische Übersicht, S. 238f. Hufeland hatte hier in einer Anmerkung auch das Meeresleuchten als Zeichen für das Vorhandensein elektrischer Strömungen im Seewasser gedeutet. Er hat an dieser Ansicht auch trotz der folgenden Kontroverse festgehalten; vgl. Über das Leuchten und die flüchtigen Bestandteile des Seewassers, als Auszeichnung der Seebäder, J.H.31 (1810) St.6, S.89ff., (wieder abgedruckt in: Kleine medizinische Schriften, Band IV, S. $340 \mathrm{ff}$.).

144. Vgl. Dissertatio inauguralis sistens usum vis electricae in Asphyxia, experimentis illustratum, abgedruckt in: Kleine medizinische Schriften, Band I, Berlin 1822, S. $1 \mathrm{ff}$.

145. Ideen über Pathogenie, S.26.

146. Ibidem S.91.

147. Сн.Е.Н.Вischoff, Über den Galvanismus und dessen Anwendung, zitiert nach K.PFEIFER, a. a.O. S.158f.

148. Enchiridion, S.354.

149. Vgl. S. 200.

150. Praktische Übersicht, S.138f. und 237.

151. Ibidem S.164. 
152. Ibidem S.73.

153. Vgl. Anm.141.

154. Praktische Übersicht, S.73f.

155. Ibidem S. 20.

156. Vgl. M.NeUburger, a.a. O., S.93.

157. Vgl. Mein Begriff von der Lebenskraft, J.H.6 (1798) St. 5, S. 785 ff. (wieder abgedruckt in: Kleine medizinische Schriften, Band II, S.344ff.); siehe auch M.NeUBURGer, a. a.0., S.91.

158. B.M.Lensch, Geschichte der Balneologie, Hydroposie und Pegologie, Würzburg 1863, S.212f. 